This PDF is a selection from a published volume from the National Bureau of Economic Research

Volume Title: Investigations in the Economics of Aging

Volume Author/Editor: David A. Wise, editor

Volume Publisher: University of Chicago Press

Volume ISBN: 0-226-90313-3; 978-226-90313-2 (cloth)

Volume URL: http://www.nber.org/books/wise11-2

Conference Date: May 5-8, 2011

Publication Date: May 2012

Chapter Title: The Value of Progress against Cancer in the Elderly

Chapter Authors: Jay Bhattacharya, Alan M. Garber, Matthew Miller, Daniella Perlroth

Chapter URL: http://www.nber.org/chapters/c12439

Chapter pages in book: (p. $203-233$ ) 


\title{
The Value of Progress against Cancer in the Elderly
}

\author{
Jay Bhattacharya, Alan M. Garber, \\ Matthew Miller, and Daniella Perlroth
}

\subsection{Introduction}

Cancer remains one of the most common causes of death in the elderly despite large investments in early detection and better treatments over the last forty years (Cutler 2008). The number of cancer deaths in the United States began falling in the early 2000s - the first decline since reliable cancer mortality statistics were collected (Lenzer 2006). This trend of improved overall survival, including for the most common types of cancers-breast, prostate, lung and colorectal cancers - continued at least through the end of the last decade, when the latest national statistics were available (National Cancer Institute 2010).

Jay Bhattacharya is associate professor at the Center for Primary Care and Outcomes Research (PCOR), Stanford University School of Medicine, and a research associate of the National Bureau of Economic Research. Alan M. Garber is provost of Harvard University, the Mallinckrodt Professor of Health Care Policy at Harvard Medical School, professor of economics in the faculty of arts and sciences, and professor of public policy in the Harvard Kennedy School of Government. He is also a research associate of the National Bureau of Economic Research. Matthew Miller was a research associate at the Center for Health Policy/Center for Primary Care and Outcomes Research (CHP/PCOR) at Stanford University and a medical student at the University of California, Los Angeles, during the course of this work. He is currently an engagement manager with McKinsey and Company's southern California office. Daniella Perlroth is an instructor at the Center for Health Policy/Center for Primary Care and Outcomes Research (CHP/PCOR) at Stanford University.

This work was supported by National Institute on Aging Grants AG17253 and AG05842, K02-AG024237, and by National Cancer Institute Grant HS09121. We thank participants at the American Economics Association meetings and at the National Institute on Aging Roundtable at the Center for Medicare and Medicaid Services for helpful suggestions. We especially thank Amitabh Chandra and the rest of the participants at the 2011 NBER Program Project meeting on aging. We also thank Grant Miller and Nicole Smith for their suggestions and comments. For acknowledgments, sources of research support, and disclosure of the authors' material financial relationships, if any, please see http://www.nber.org/chapters/c12439.ack. 
Before these recent gains, some analysts questioned whether substantial progress had been made against cancer despite numerous innovations in methods to prevent, detect, and treat the conditions (Bailar and Gornik 1997). Among the innovations were policies to discourage cigarette smoking, campaigns to promote screening for cervical, breast, and colorectal cancer, better imaging technologies, and advances in treatment with new chemotherapeutics and next-generation radiation therapy (e.g., intensitymodulated radiation therapy). More recent treatment innovations have included costly antibody and immune therapies, such as bevacizumab (Avastin) for colorectal cancer and sipuleucel-T (Provenge) for prostate cancer (Chambers and Neumann 2011).

Because cancer is prevalent in the elderly, and Medicare expenditure growth is a critical policy challenge (2008), it is likely that costly treatment advances will increasingly need to demonstrate value (Elkin and Bach 2010). One approach for assessing the value in technology advances has been the application of cost-effectiveness analysis to medical decision making (Garber and Phelps 1997; Owens et al. 2011). Other credible definitions of value are essentially variations of the cost-benefit framework (Porter 2010). Costeffectiveness analysis of individual physician-patient decision making has made advances in research methodology and is now an accepted methodology in the medical literature to guide medical decision making.

The evidence that outcomes have improved for some medical conditions is clear. Over the past twenty-five years, mortality from heart disease has declined substantially (Rodriguez et al. 2006), effective treatment for HIV disease has become available, the microbial basis for peptic ulcers has been elucidated and resulted in more effective treatment, and new classes of drugs have improved care for serious psychiatric, cardiac, and rheumatologic diseases. Some economists have estimated that new medical technologies have led to large gains in survival and health, with extraordinary economic value (Murphy and Topel 2003, 2005). According to one analysis, "between 1970 and 2000 increased longevity added about $\$ 3.2$ trillion per year to national wealth" (Murphy and Topel 2006, 871).

These health gains have come at a time of unsustainable growth in health expenditures, particularly for Medicare (Medicare Trustees 2008). Consequently, a debate has arisen about whether these benefits are worth the costs they require. Some have argued that outcomes for myocardial infarction patients have improved so greatly that increased expenditures on these new technologies are justified (Cutler 2004; Cutler and McClellan 2001) Others have argued that many new technologies raise costs without conferring significant health benefits (Meltzer 2003; Siegler, Weisfeld, and Cronin 2003). Indeed, the medical care subsidy embedded in health insurance and other market distortions create incentives for the adoption of new medical technologies even when their benefits exceed their costs (Fuchs and Garber 2003, 
1990). Further, there is evidence from the literature on regional variations in the standards of medical care that the early adoption of new medical technology may not translate into improved health (Skinner, Staiger, and Fisher 2006).

We extend these attempts to assess the value of medical progress at the population level by matching changes in survival for cancer with changes in spending for those conditions. This has been done in the past, most notably by Cutler and McClellan (2001), who evaluated aggregate outcomes and expenditures on Medicare patients and found that advances in acute heart attack care and cataract surgery were highly likely to have met conventional cost-effectiveness criteria in the past. In their evaluation of breast cancer care in the 1990s, they were unable to rule out cost-ineffective advances in breast cancer treatment with their approach. Thus, the aggregate population approach to estimating value in medical progress has been attempted before, and could be useful to policymakers, for example, when considering changes to public health programs or prioritizing population health investments.

With this research, we compare improvements in overall survival after a cancer diagnosis with changes in the cost of medical care for the cancer patient. We begin by evaluating the relative contribution of changes in diagnosis and treatment to changes in survival after a diagnosis of breast, prostate, lung, or colorectal cancer, separately for men and women. We combine these estimates (survival, expenditures) into a traditional cost-effectiveness approach by estimating the marginal cost-effectiveness of improvements in the detection and treatment of cancer for two periods: 1986 to 1994 and 2000 to 2004.

We run two versions of this analysis. In one, we measure trends in survival and expenditures among diagnosed cancer patients, not adjusted for trends in staging. These trends presumably represent changes in these outcomes due to changes in treatment technology and in diagnostic and staging technology. In the second version, we present survival and expenditure trends holding fixed staging at a fixed date. We do this because diagnostic improvements will influence survival by shifting diagnosis to less advanced stages of disease. These stage-adjusted trends presumably represent changes in these outcomes due to changes in treatment technology alone, though there are some important subtleties in this interpretation which we will discuss shortly.

We study four cancers (prostate, breast, colorectal, and lung) that together account for over half of all new cancer cases in the elderly, and for which the elderly represent 70 percent of new cases (Potetz and DeWilde 2009). Understanding the relative effectiveness of early diagnosis and treatment advances for these cancers is important in deciding where increasingly limited anticancer resources should be allocated. 


\subsection{Methods}

We evaluate the two periods of analysis using similar methodologies for assessing increases in expenditures and changes in survival for cancer patients. The first period (1986-1994) represents a time of screening innovation and higher rates of mammography, Prostate-Specific Antigen (PSA) testing, and colon cancer screening exams. The second period (2000-2004) had mostly flat rates of screening for breast (with a slight decline starting in 2003), with modest increases in the rates of colorectal cancer screening and PSA screening for prostate cancer (National Cancer Institute 2010). During this second period, we evaluate breast, prostate, colorectal, and lung cancer survival and expenditure changes. We refer to each cancer type and gender diagnosed during each period separately as a cohort.

\subsubsection{6 to 1994 Data}

Our data during this period are drawn from the 20 percent random sample of the Centers for Medicare and Medicaid Services Health Insurance Skeleton Eligibility Write-off (HISKEW) files, the basic data set used to track Medicare eligibility. Only elderly men and women enrolled in the traditional (fee-for-service) Medicare program are included, because accurate diagnostic information is not available for Medicare managed care enrollees in claims files. Beneficiaries who are eligible based upon disability or end-stage renal disease are also excluded.

We identified all men and women with a diagnosis of breast, prostate, or colorectal cancer, using diagnosis and procedure fields from each claim, noting the first date that a diagnostic code for cancer was recorded for the individual. These claims were then linked to all subsequent claims for these patients.

From this final sample, we calculated Medicare expenditures and survival in the five years after the initial diagnosis year for each cohort of newly diagnosed cancer patients. We characterized the experiences of beneficiaries with breast cancer, prostate cancer, and colorectal cancer, the latter separately for men and women.

Early reports from the Institute of Medicine suggested that using claims to infer diagnosis is error-prone (Institute of Medicine 1977), while more recent reports suggest that error rates are lower (Fisher, Wennberg et al. 1994; Fisher, Whaley et al. 1992; Mark 1994; McBean, Warren, and Babish 1994). As one test of reliability, we compared mortality rates in our sample to mortality in the Surveillance, Epidemiology, and End-Results (SEER) database, which contains detailed information on a nationally representative sample of US cancer patients. We found that mortality rates for breast, prostate, and colorectal cancer in the two samples matched closely.

We calculated one-year, three-year, and five-year survival probabilities for each group using date of first diagnosis and date of death. We estimated 
patient life expectancy by fitting these survival data to standard statistical models of mortality. We also calculated medical expenditures in each year following initial cancer diagnosis. We calculate the net present value (NPV) of five years of Medicare expenditures after cancer diagnosis, including the diagnosis year. We include all medical care costs, not only cancer-related costs, the appropriate approach to cost-effectiveness analysis under most circumstances (Garber and Phelps 1997). Annual expenditures were calculated as the sums of expenditures for all claims over each twelve month period after the diagnosis, from anniversary to anniversary of the diagnosis date. Expenditures include Part A (inpatient hospital), Part B (outpatient), physician, home health, and hospice services. These totals include chemotherapy and inpatient pharmaceutical expenditures but exclude outpatient prescription drugs, which Medicare did not cover during the periods in question. Claims in each category were available for everyone in the 20 percent sample, except for physician services, which were only available for a onequarter random subset of the 20 percent sample. We adjusted all results for inflation, reporting results in constant year 2000 US dollars.

The SEER data were used to adjust the survival and expenditure estimates for age-specific trends in staging, which is not recorded in claims files. We merged SEER data to the appropriate Medicare claims by cohort and age and calculated estimates of cohort-specific five-year survival and Medicare expenditures adjusted for age and stage at diagnosis. We performed nonlinear regressions of five-year survival and expenditures (separately) on flexible functions of age and cohort as well as on the proportion of each age-cohort diagnosed at the various stages of disease (Garber and MaCurdy 1993).

\subsubsection{0 to 2004 Data}

For this period, our basic approach was the same as the aforementioned, with a few modifications. First, we used the Surveillance, Epidemiology, and End Results (SEER) Program case files directly linked to Medicare claims data. The main advantage of this directly linked data is that we are able to calculate stage-specific survival and expenditure trends, rather than just stageadjusted trends. We selected newly diagnosed patients with breast, prostate, colorectal, and lung cancer from 2000 to 2004 based on the SEER files. The SEER incorporates seventeen geographic regions including Seattle/Puget Sound, California, Utah, New Mexico, Alaska, Hawaii, Louisiana, Iowa, Kentucky, Atlanta and rural Georgia, Detroit, New Jersey, and Connecticut. Table 6.1 reports the sizes of each cohort based on year of diagnosis. We obtained linked SEER-Medicare claims data through 2009 to ensure five years minimum of claims data for all patients. Similar exclusionary criteria to the aforementioned period were used, including enrollment in Part $\mathrm{C}$ at any time during this period. We evaluated endpoints of one-, three-, and five-year survival probabilities and mean health expenditures adjusted for dummy stage, dummy age, and year of diagnosis (excluding outpatient phar- 
Table 6.1

Cancer cohort sizes (2000-2004)

\begin{tabular}{lrrrrrr}
\hline & 2000 & 2001 & 2002 & 2003 & 2004 & Total cases \\
\hline Prostate & 12,175 & 12,944 & 13,549 & 12,258 & 19,119 & 70,045 \\
Breast & 15,069 & 15,889 & 16,188 & 15,414 & 15,286 & 77,846 \\
Lung-Men & 9,367 & 9,889 & 10,220 & 10,604 & 9,679 & 49,759 \\
Lung-Women & 7,550 & 8,239 & 8,768 & 9,025 & 8,603 & 42,185 \\
Colorectal-Men & 7,293 & 7,540 & 7,756 & 7,694 & 7,267 & 37,550 \\
Colorectal-Women & 8,253 & 8,330 & 8,629 & 8,358 & 7,754 & 41,351 \\
\hline
\end{tabular}

maceutical, or Part D expenditures). For this time period only, we calculated five-year survival rates by stage of disease for each annual cancer cohort. The calculation of stage-specific survival rates is not possible with the data from the previous time period.

We calculated life expectancy using hazard rates calculated from the one-, three-, and five-year survival probabilities by assuming the hazard rate was constant over time. We need such an assumption for life expectancy calculations since we do not observe the whole sample through its whole life span.

We follow the approach of Cutler and McClellan in calculating the marginal cost-effectiveness ratios of Medicare expenditures on medical care for cancer (Cutler and McClellan 2001). These should not be interpreted as cost-effectiveness ratios corresponding to any specific clinical intervention or policy. These are calculated by dividing the change in the NPV of Medicare expenditures between two periods by the change in life expectancy between those same periods; the interpretation as cost-effectiveness ratios is based on an assumption that changes in treatment and screening for patients during this period are solely responsible for changes in expenditures and survival. The stage-adjusted numbers can be similarly interpreted (under an analogous assumption) as cost-effectiveness ratios corresponding to changes in treatment regimens over the specified periods. We perform these calculations annually and for the overall periods 1986 to 1994 and 2000 to 2004.

For the analysis of 2000 to 2004 data, we included a terminal value for the cost of medical care beyond the five years of Medicare claims. We did this for breast, prostate, and colorectal cancer patients because their average life expectancy was greater than the five years of expenditure data. We estimated the terminal cost component by assuming that observed average medical costs in the fifth year after diagnosis continue for each cohort. We adjusted future annual expenditures for inflation by applying the medical Consumer Price Index (CPI) in 2000 (4.1 percent) for the remaining average years of life expected based on the calculation from observed five-year survival. We then discounted all years of expenditures back to 2000 US dollars at 5 percent and summed to estimate the final terminal component for medical expenditures 
past the five years of data (and for the remaining average life expectancy left for each cohort).

\subsubsection{Cancer Staging Systems}

For stage evaluation, we rely on data from Surveillance Epidemiology and End Results (SEER) Program Registry. The SEER data report staging using two different, though related, staging classification systems. The first is a historical staging system that relies on a consistent staging system definition that did not change during the years we analyze. The second system is called the American Joint Committee on Cancer (AJCC) staging system, which is periodically updated. The AJCC was founded by the American College of Surgeons in 1959 to establish a national standard for cancer staging (Fleming 2001). Both systems rely on the "TNM" system, according to the extent of the primary tumor "T," the involvement of lymph nodes "N," and the presence of distant or metastatic, "M," disease. Since 1982, the TNM criteria have become the single major approach to cancer staging throughout the world (Hutter 1984). Every five years, the AJCC makes minor updates to the TNM staging manual to reflect interim changes in the management of cancer diagnosis and treatment.

\subsection{Results}

\subsubsection{Age and Survival Trends}

For each period, we investigated trends in survival and age at diagnosis. Figure 6.1 shows that age-adjusted cancer survival rates increased substantially between 1986 and 1994 for breast and prostate cancer patients. This is true whether survival rates are measured at one, three, and five years after diagnosis, and the change between 1986 and 1994 is statistically significant at $p<0.01$. Men with colorectal cancer experienced a small and statistically insignificant rise in age-adjusted survival, while survival in women with colorectal cancer did not increase. That cancer deaths and age-adjusted survival rates can increase at the same time should not be surprising: the size of the elderly population grew over this period and falling mortality rates from competing causes of death such as heart disease also left a larger pool at risk for cancer. During the later period, 2000 to 2004 (fig. 6.2), age- and stage-adjusted three- and five-year survival increased for all cancer cohorts, again with the greatest increases in prostate and breast cancer, followed by men and women with lung cancer and women with colorectal cancer. Men with colorectal cancer had only slight survival gains.

Figure 6.3, which plots the probability that new diagnoses are found at an early stage adjusted for age, shows a shift toward earlier diagnosis at a time when surveillance efforts, such as mammography, colonoscopy, sigmoidoscopy, and prostate cancer screening were increasingly used in the Medicare 


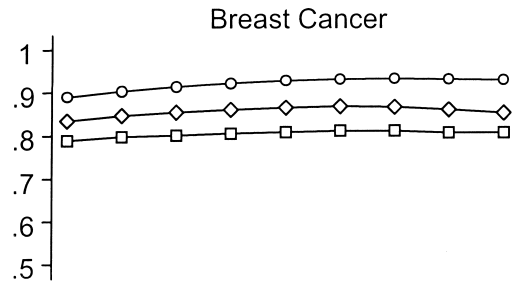

Prostate Cancer

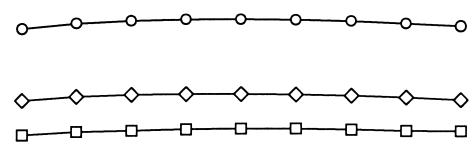

Colon Cancer; Male

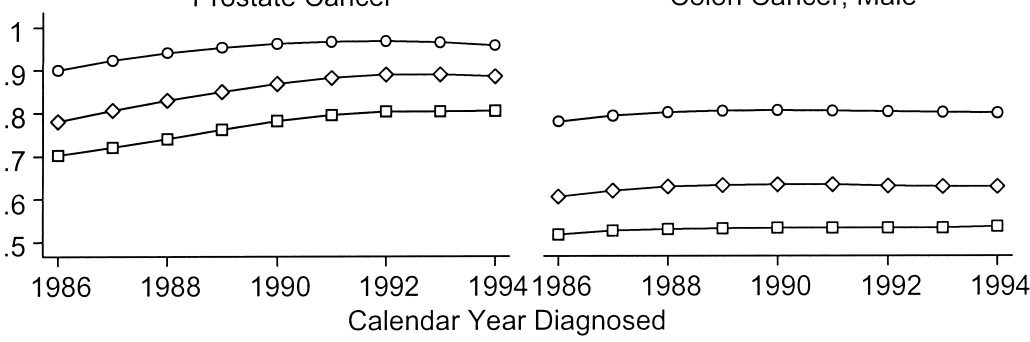

$\longrightarrow$ 1-year survival $\longrightarrow$ 3-year survival $\longrightarrow$ 5-year survival

Fig. 6.1 Age-adjusted survival probabilities by year of diagnosis (1986-1994)

Notes: Trends in survival probabilities were smoothed; the figures show predicted survival probabilities, adjusted for age, for 65 -year-olds in each year.

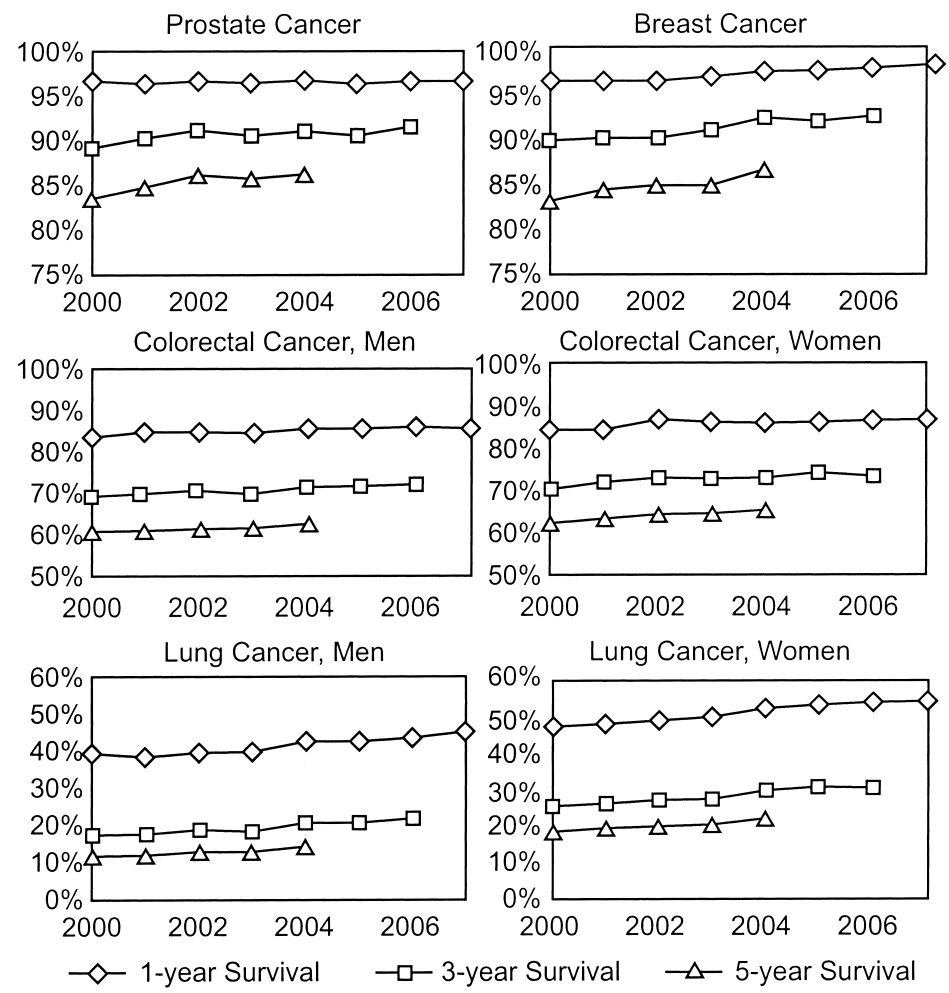

Fig. 6.2 Age-adjusted survival probabilities by year of diagnosis (2000-2004)

Note: Survival adjusted for age 65 to 69 -year-olds with stage mix of 2000 cohorts. 


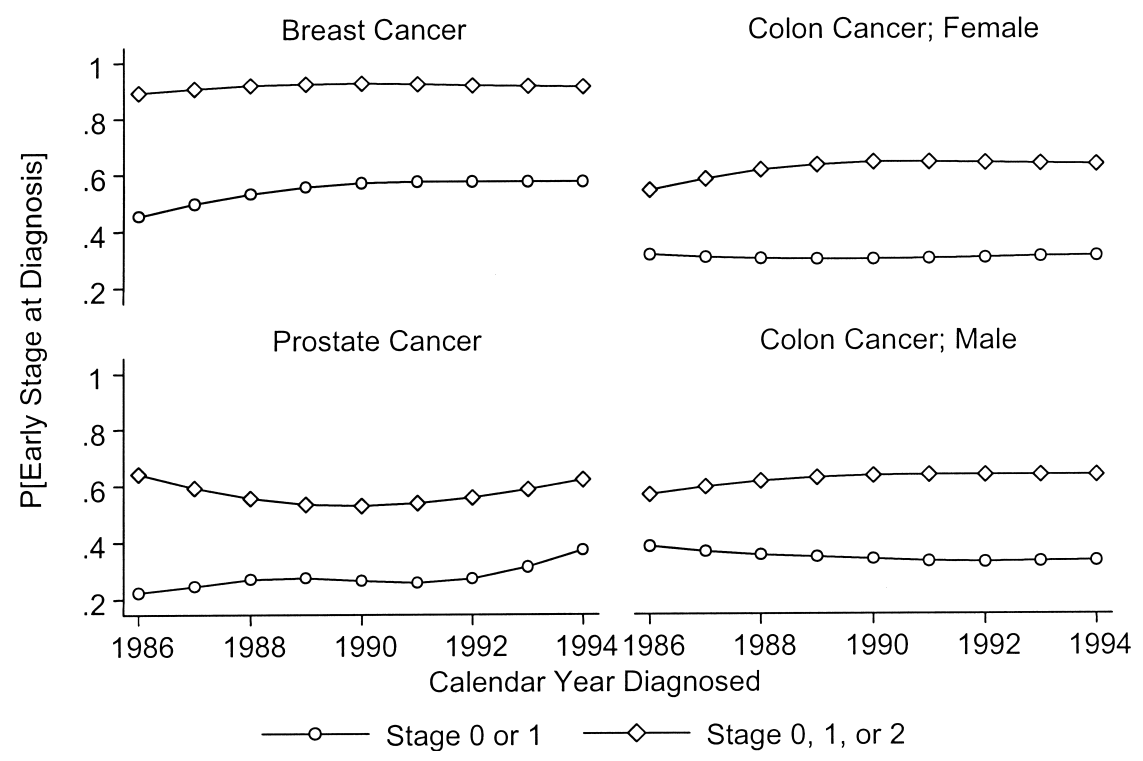

Fig. 6.3 Age-adjusted trends in stage at diagnosis (1986-1994)

Notes: Trends in survival probabilities were smoothed; the figures show predicted survival probabilities, adjusted for age, for 65 -year-olds in each year.

population (1986-1994). In 1986, 45 percent of sixty-five-year-old women diagnosed with breast cancer had stage 0 or 1 cancer. By 1994, 60 percent had stage 0 or 1 breast cancer. For prostate cancer patients the probability of a stage 1 diagnosis for a sixty-five-year-old male increased from 20 percent to 40 percent between 1986 and 1994. For colorectal cancer patients, though the probability of finding a stage 1 cancer remained flat through the period, the probability of finding either a stage 1 or 2 cancer went up for both men and women. For the later period (fig. 6.4), the portion of early stage cancers for lung, colorectal, and breast cancer changed little, reflecting small increases in colorectal cancer screening over this period, and flat to declining rates in the use of mammography (an estimated 4 percent decline in mammography occurred between 2000 and 2005; Breen et al. 2007). The proportion of early stage prostate cancer did grow, reflecting continued adoption and increased frequency of PSA testing for cancer screening during this period. During this period, Medicare also began reimbursing providers for annual prostate screening examinations (digital rectal examinations and PSA blood tests) starting in 2000, and routine screening colonoscopies every ten years for individuals with normal cancer risk starting in 2001 (Freeman et al. 2002).

The effect of shifts in diagnosis on cancer survival can be seen in figure 6.5 , which compares the change in age-adjusted five-year survival probabilities from 1986 levels when those probabilities are and are not adjusted for 

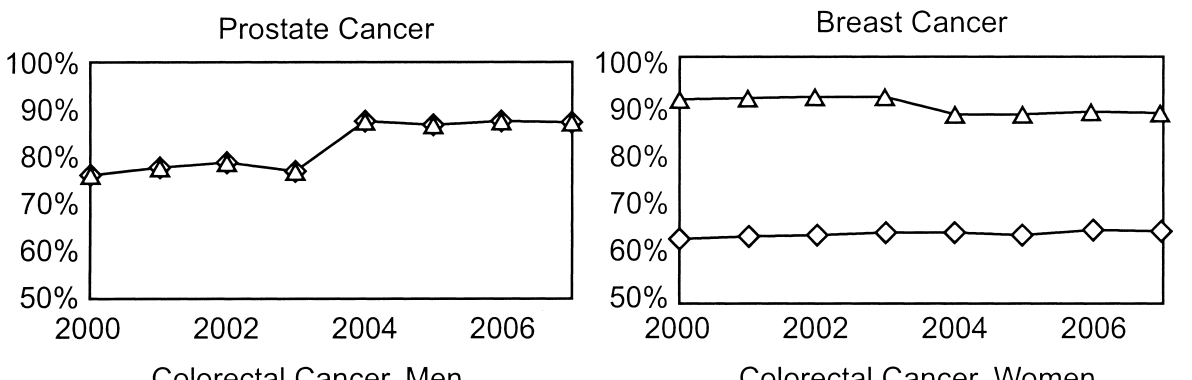

Colorectal Cancer, Men
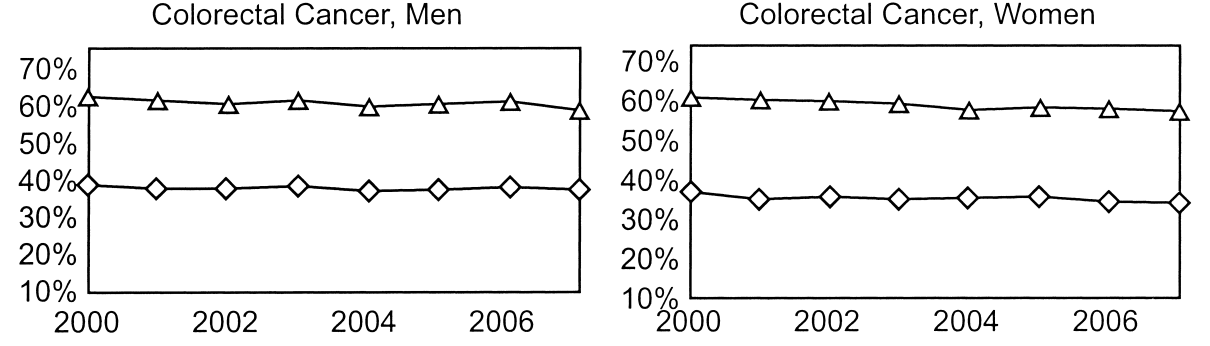

Lung Cancer, Men

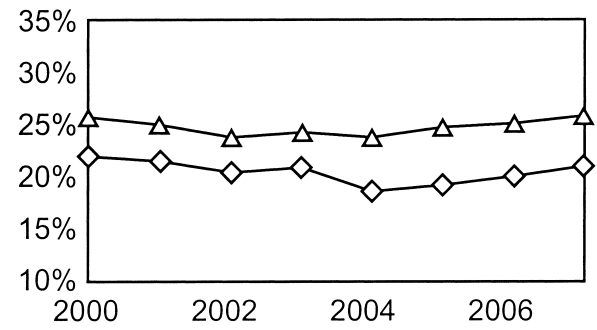

Lung Cancer, Women

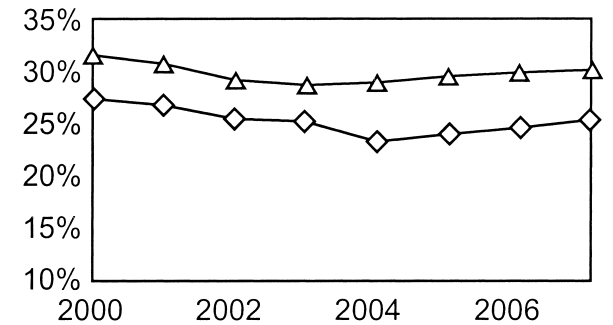

${ }^{\star}$ Adjusted for age 65 year of age at diagnosis.

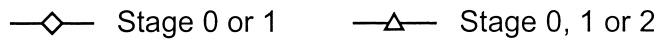

Fig. 6.4 Age-adjusted trends in stage at diagnosis (2000-2004)

Note: Adjusted for age 65, year of age at diagnosis.

staging trends. Stage adjustment has a dramatic effect on estimated breast cancer survival. A patient diagnosed in 1994 had a 10 percentage point lower probability of five-year survival than a patient with the same stage and at the same age diagnosed in 1986; survival rates not adjusted for stage show little improvement over the same period. By contrast, for men with colorectal cancer, stage-adjusted survival probability improved substantially between 1986 and 1994. Stage-adjustment has little effect for either prostate or colorectal cancer in women during this period.

During the 2000 to 2004 period, stage-adjusted five-year survival increased 


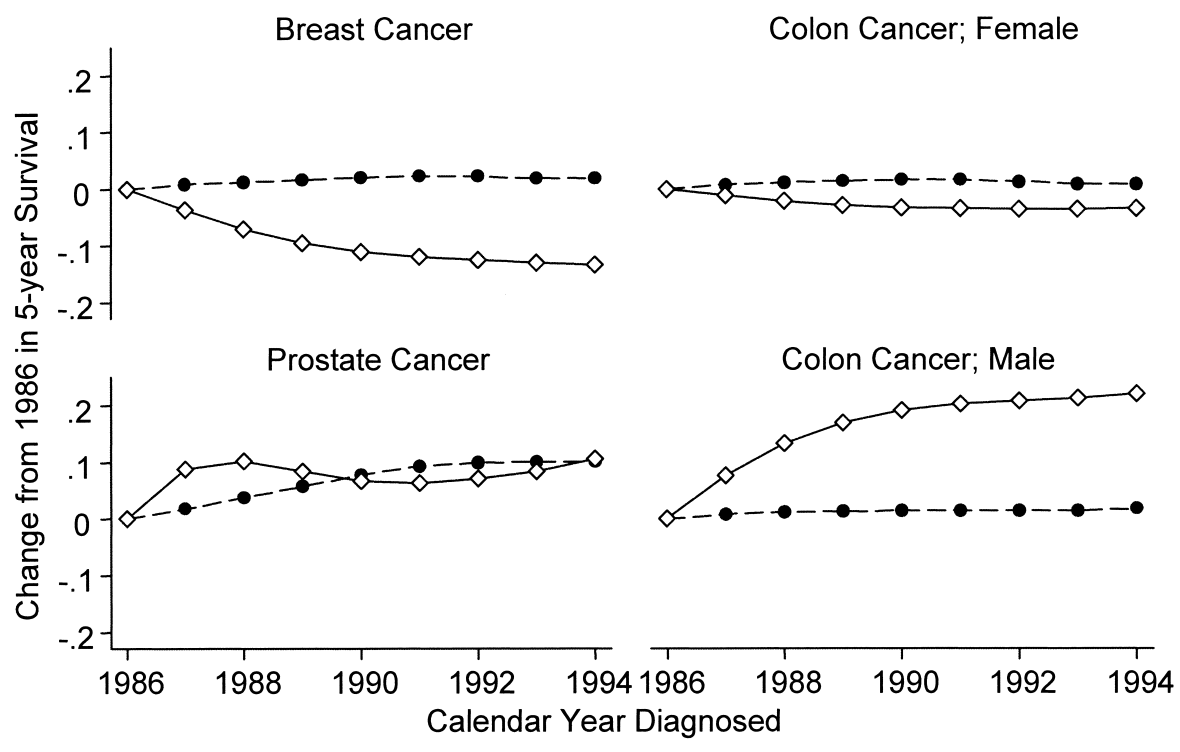

- - - - Not adjusted for staging trends $\_$Adjusted for staging trends

\section{Fig. 6.5 Adjusted average changes in 5-year survival (1984-1996)}

Notes: Trends in survival probabilities were smoothed; the figures show predicted survival probabilities, adjusted for age, for 65 -year-olds in each year.

each year for all cancer cohorts compared to 2000 survival (fig. 6.6). The survival gains during this period were greater for women, with a 3 to 4 percent absolute gain in five-year survival for all cancer cohorts adjusted for stage and age of disease between 2004 and 2000, compared to a 2 to 3 percent increase for men during the same period.

\subsubsection{Expenditure Trends}

Figure 6.7 shows mean Medicare expenditures by year from diagnosis (in 1986-1994). Costs were highest during the first year after diagnosis; even after adjustment for inflation, costs increased for each successive cohort. These trends were also found in the 2000 to 2004 period (fig. 6.8). We find that expenditures are highest in the diagnosis (and presumed treatment) year, fall precipitously soon after, and then grow gradually as survivors age, as has been observed in other studies. Initial year costs for the 2000 to 2004 period were greatest for colorectal cancer, followed by lung cancer, followed by breast and prostate cancer. Expenditures for men and women were roughly equal in the year following diagnosis for both colorectal and lung cancers.

Figure 6.9 plots age-adjusted expenditure trends alongside age- and stage-adjusted expenditure trends. The figure confirms the expectation that 

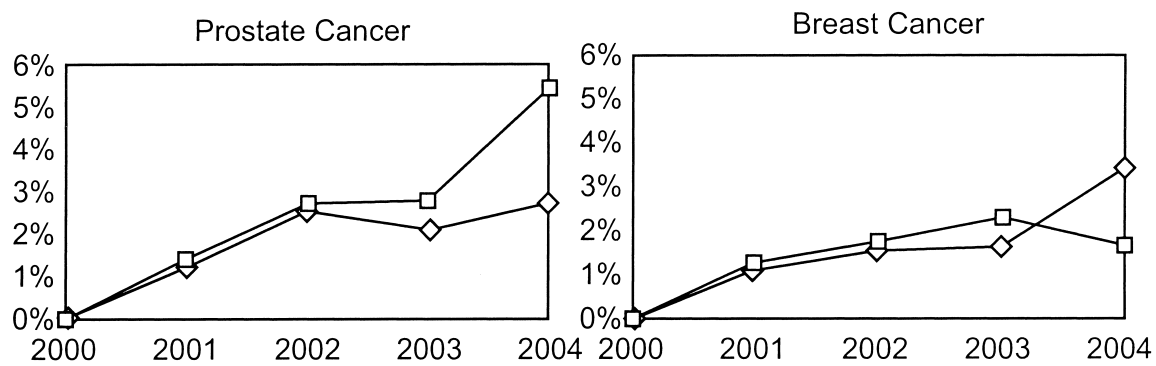

Colorectal Cancer, Men
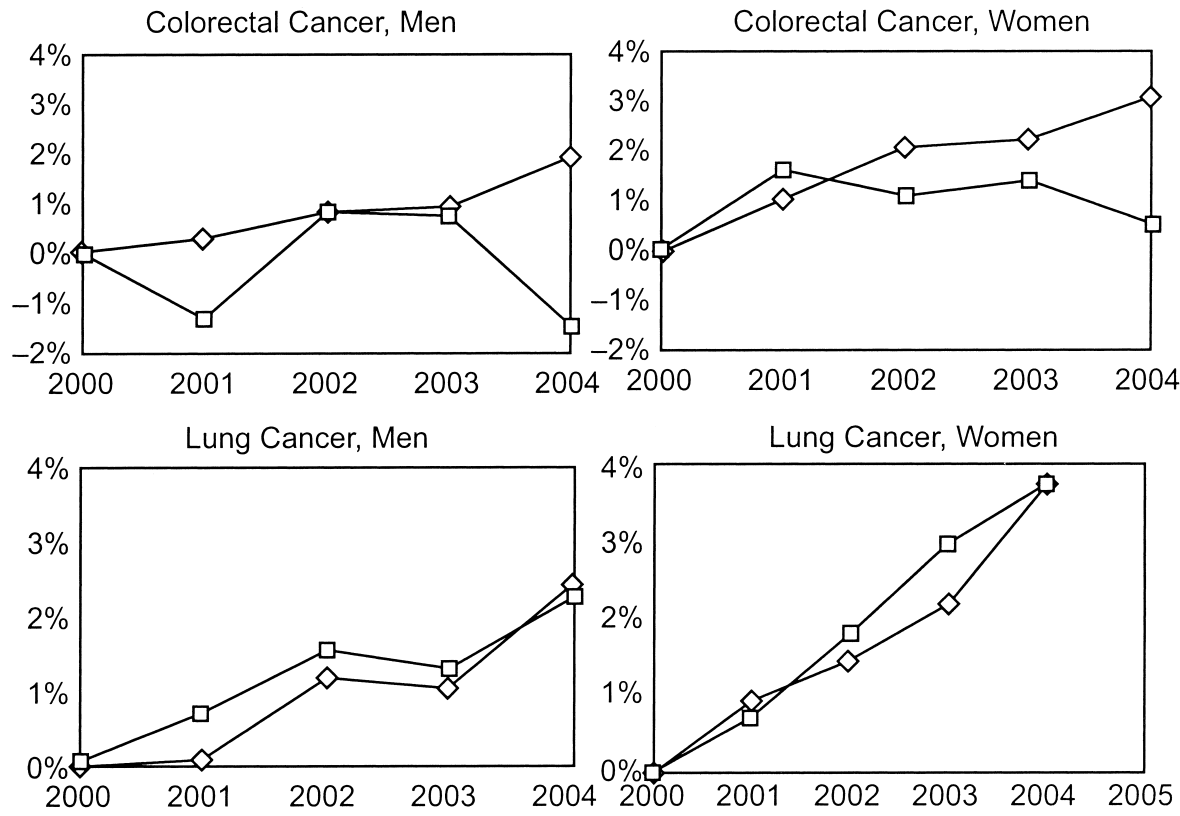

*Adjusted for age 65 years at diagnosis, staged fixed in 2000 cohort distributions.

$$
\checkmark \text { Stage Fixed } \quad \square-\text { Stage Not Fixed }
$$

Fig. 6.6 Adjusted average change in 5-year survival from 2000 (2001-2004)

Note: Adjusted for age 65 years at diagnosis, staged fixed in 2000 cohort distributions.

shifting toward earlier stages at diagnosis will reduce medical expenditures, especially during the year following diagnosis, since early stage cancer tends to be less expensive to treat than later stage cancer. In each case, the age- and stage-adjusted expenditure trends lie below the age-adjusted trends. For prostate cancer and colorectal cancer patients, the age- and stage-adjusted expenditures in the diagnosis year rose by about $\$ 10,000$ between 1986 and 1994. For breast cancer, analogous expenditures rose by about $\$ 2,000$ over the same period. 

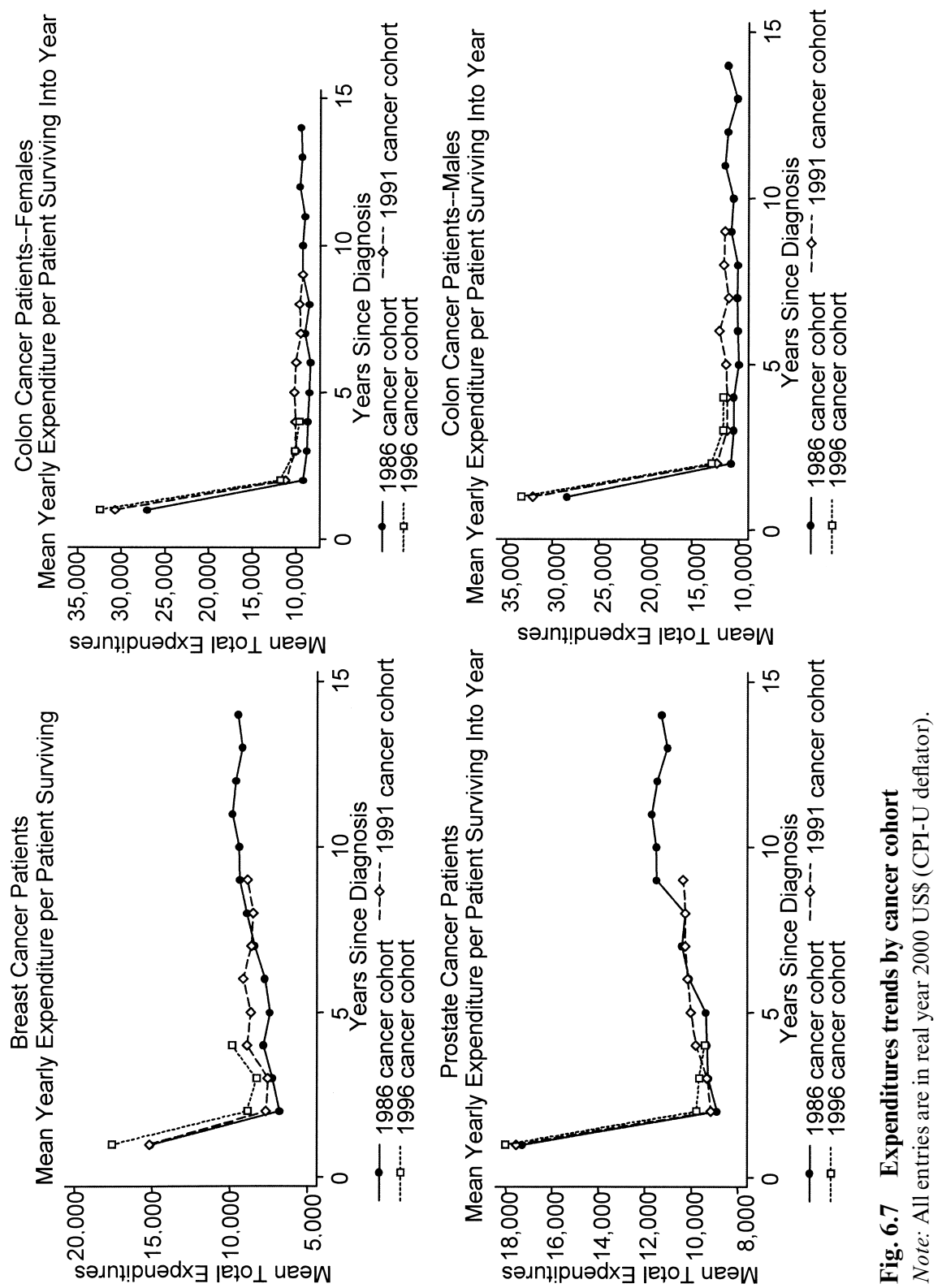

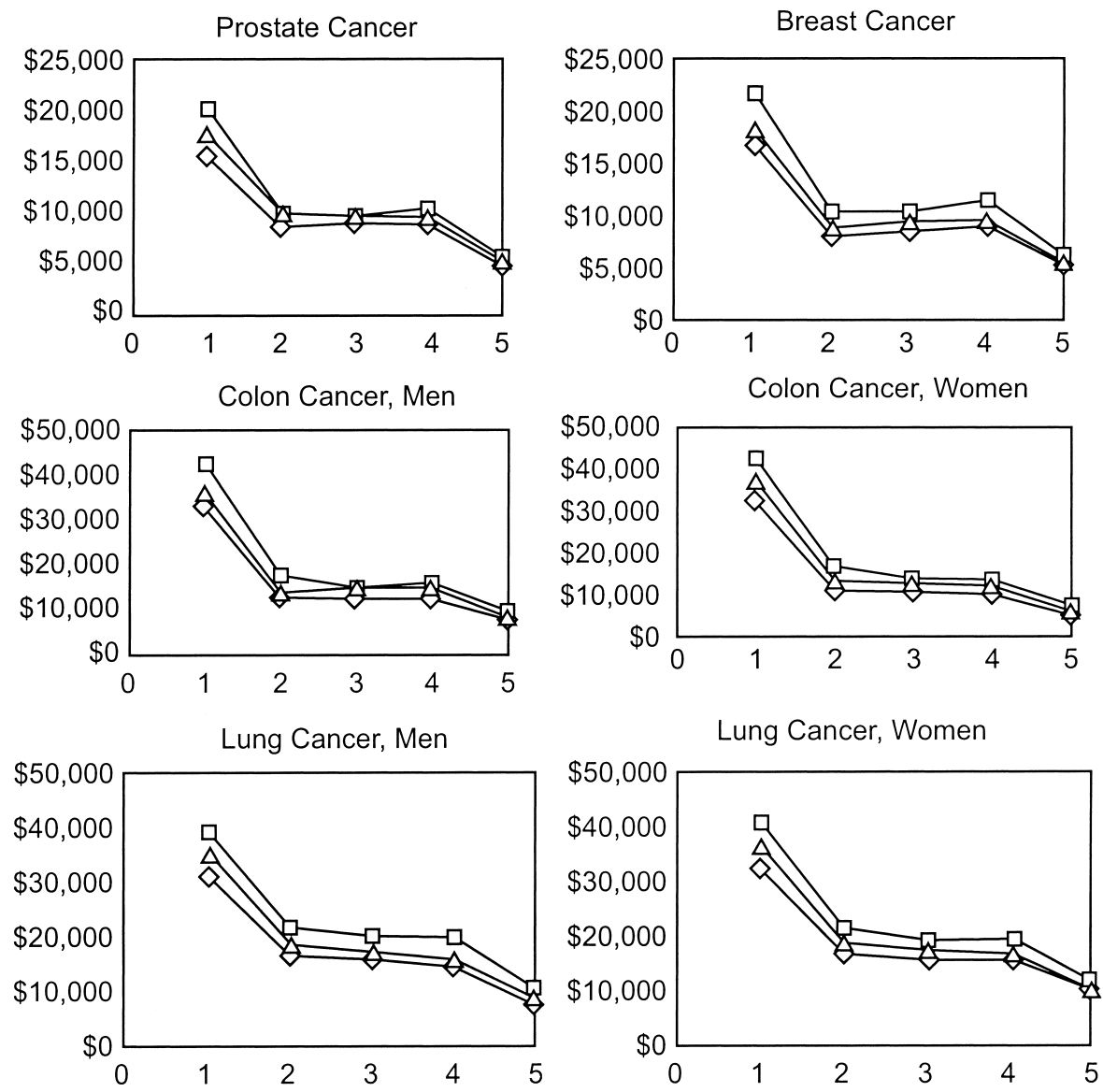

Year after Diagnosis

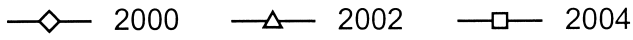

Fig. 6.8 Expenditure trends by cancer cohort (2000-2004)

Note: Expenditures adjusted to 2000 US\$ using GDP deflator.

Figure 6.10 shows the same analysis for the 2000 to 2004 cohort. This demonstrates similar trends except in the case of prostate cancer, where ageand stage-adjusted expenditure estimates are lower than age-only adjusted average expenditures. Stage- and age-adjusted expenditure growth during this period was the highest for men with lung cancer, with an NPV of $\$ 20,000$ more to treat similar age and stage of disease in 2004 over 2000. Breast and prostate cancer cases showed the lowest age- and stage-adjusted growth in expenditures during this period, with about $\$ 5,000$ more spent per case treated in 2004 for these conditions, compared with 2000. 


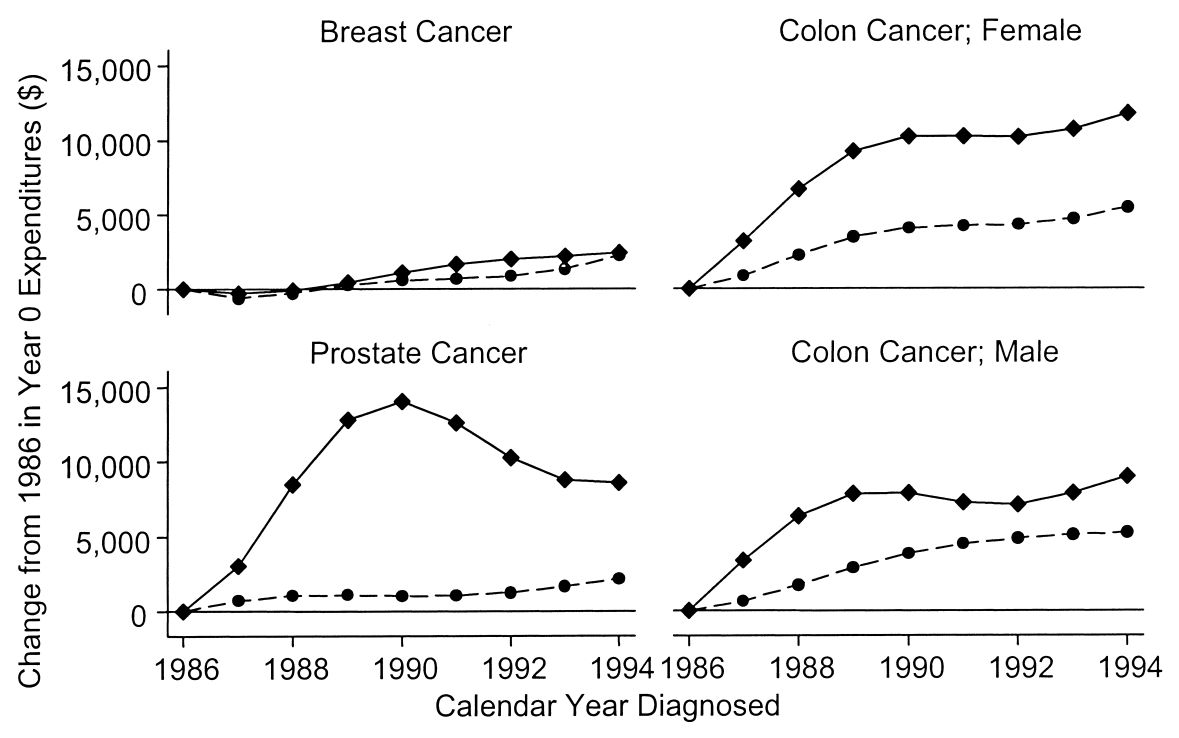

- - - Not adjusted for staging trends $\longrightarrow$ Adjusted for staging trends

Fig. 6.9 Adjusted average annual changes in expenditures (1984-1996)

Notes: Trends in survival probabilities were smoothed; the figures show predicted survival probabilities, adjusted for age, for 65 -year-olds in each year. All entries are in real year 2000 US\$ (CPI-U deflator).

\subsubsection{Incremental Cost-Effectiveness of Medical Progress for Cancer in the Elderly}

Table 6.2 shows life expectancy and the net present value (NPV) of expenditures for successive cohorts of women diagnosed with breast and colorectal cancer in the 1986-1994 period. Table 6.3 shows analogous numbers for men with prostate and colorectal cancer. Included are estimates unadjusted and adjusted for age and stage at diagnosis. These trends parallel the findings for changes in cancer outcomes over time. Unadjusted life expectancy increased for all groups, while adjusted life expectancy increased for men with prostate and colorectal cancer, fell for breast cancer patients, and stayed roughly flat for women with colorectal cancer. Unadjusted expenditures increased sharply for all four cancer groups, while adjusted expenditures changed little for men with colorectal cancer and increased for breast, prostate, and for colorectal cancer in women.

During this period, advances in medical care of patients with prostate cancer cost an additional $\$ 44,466$ when diagnosed in 1994 as compared with 1986, and these costs were associated with an incremental cost per life-year gained of $\$ 13,500$ adjusted for changes in age and stage of disease. For colorectal cancer in women, it actually cost less to treat a case in 1986 

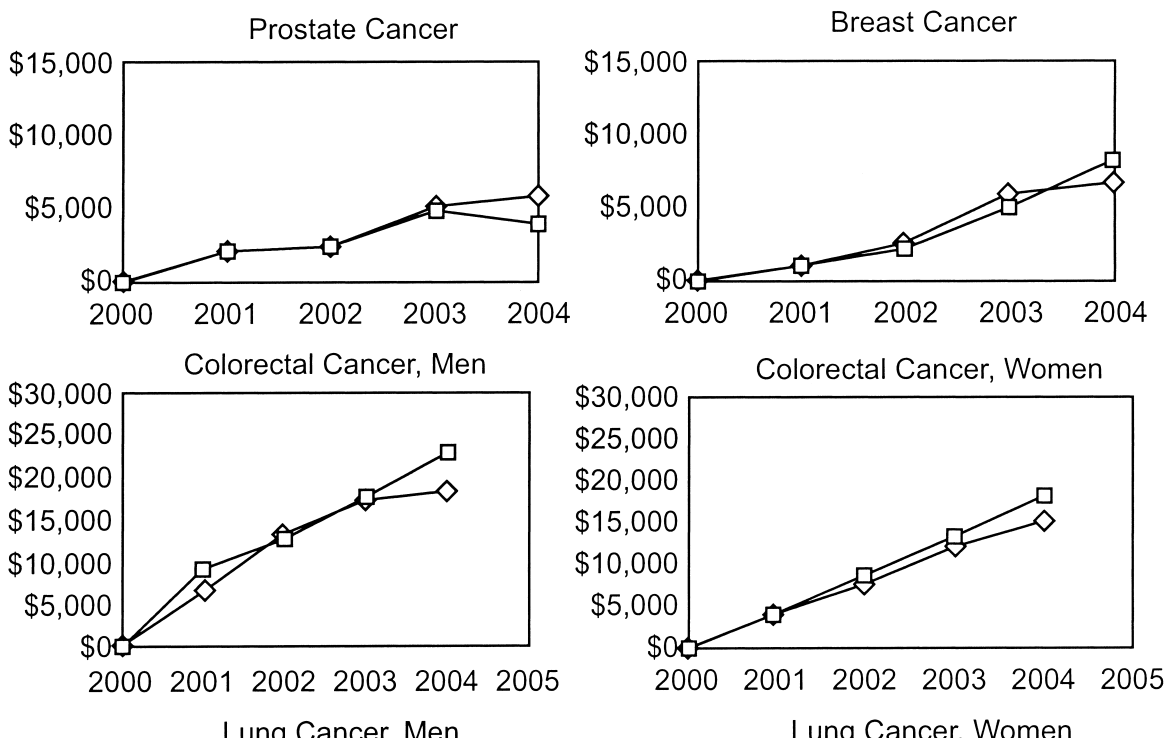

Lung Cancer, Men
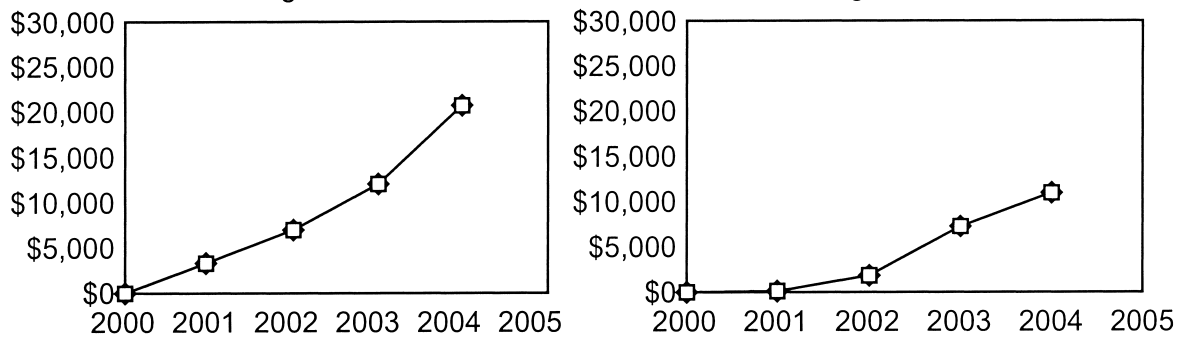

${ }^{*}$ Change in 5-year NPV from year of diagnosis compared to 2000. These show predicted probabilities for 65-69 year olds with stage proportions fixed at 2000 levels. All expenditures are in 2000 US dollars.

\section{$\checkmark$ Stage Fixed $\quad \square-$ Stage Not Fixed}

Fig. 6.10 Adjusted average annual changes in expenditures (2000-2004)

at $\$ 43,409$, as compared to care in 1994 costing $\$ 68,870$, and was associated with an estimated loss of 0.6 years of life (when diagnosed in 1994 versus 1986). Breast cancer improvements in care cost an additional $\$ 16,600$ in 1994 compared with 1986, and resulted in an age- and stage-adjusted loss of 2.6 life-years.

Tables 6.4 and 6.5 show life expectancy, five-year costs, and terminal lifetime costs of medical care for patients in the 2000 to 2004 period. These results show the greatest increase in life expectancy for patients with breast and prostate cancers, at 7.6 and 6.1 years, respectively, of additional life 


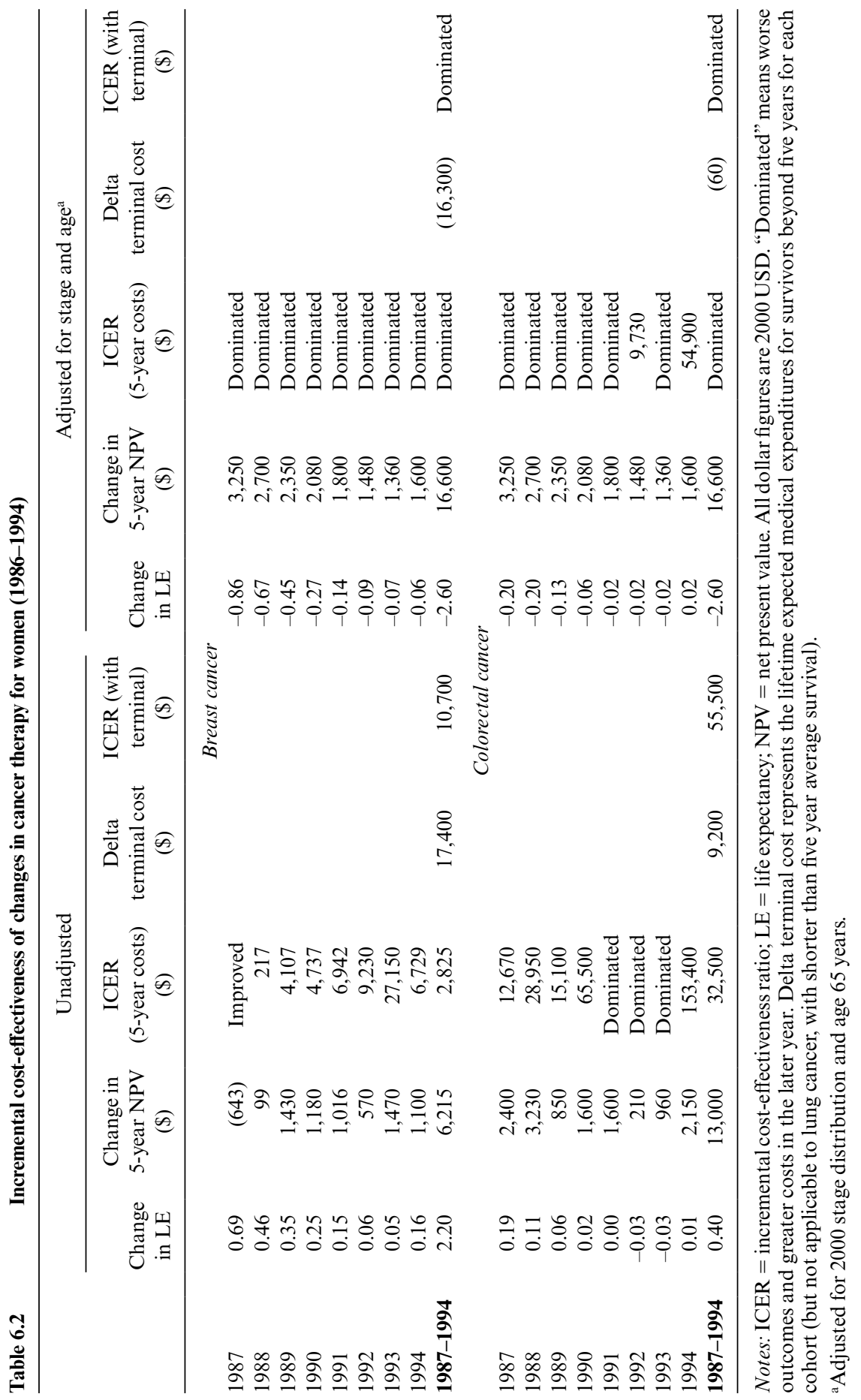




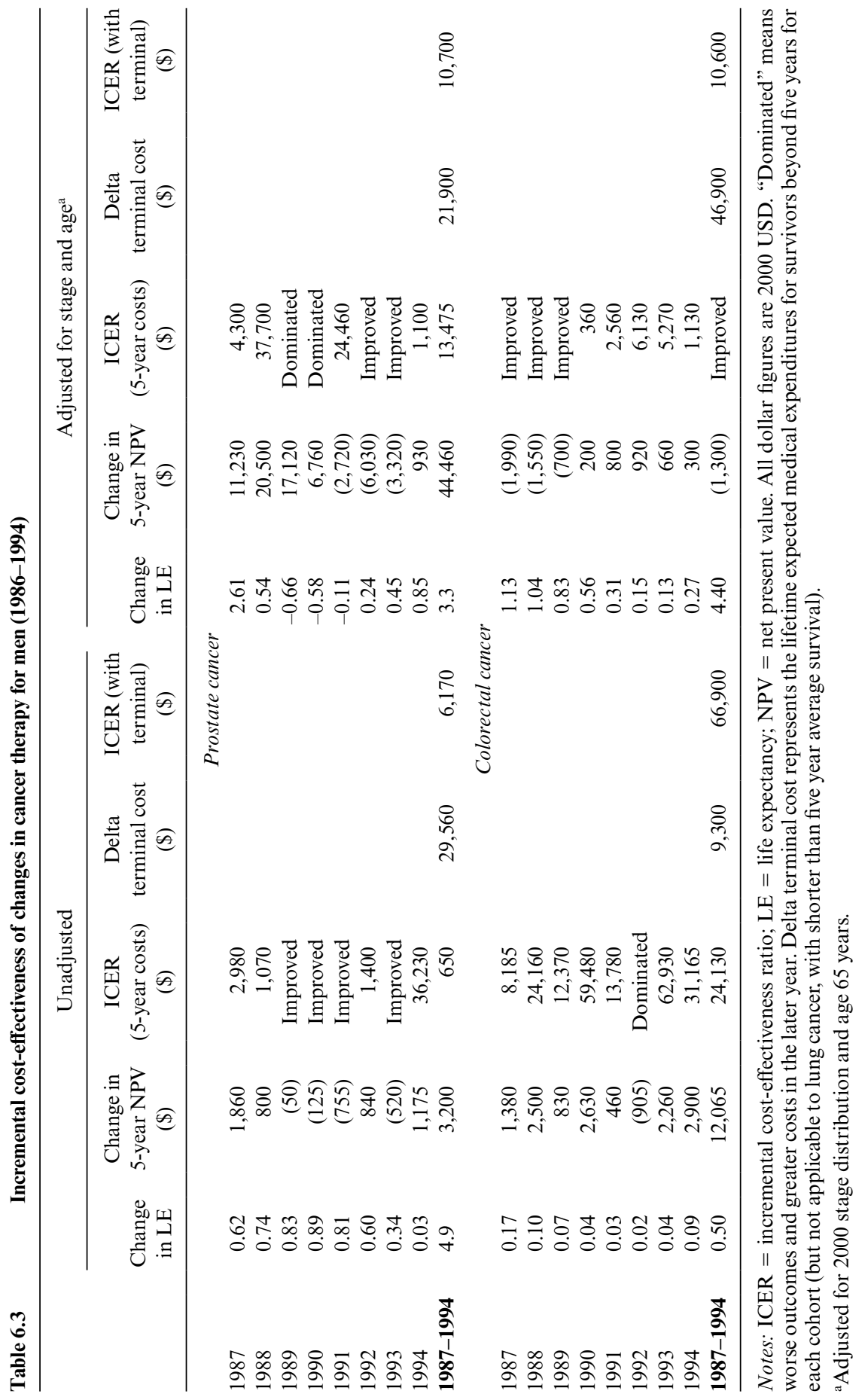




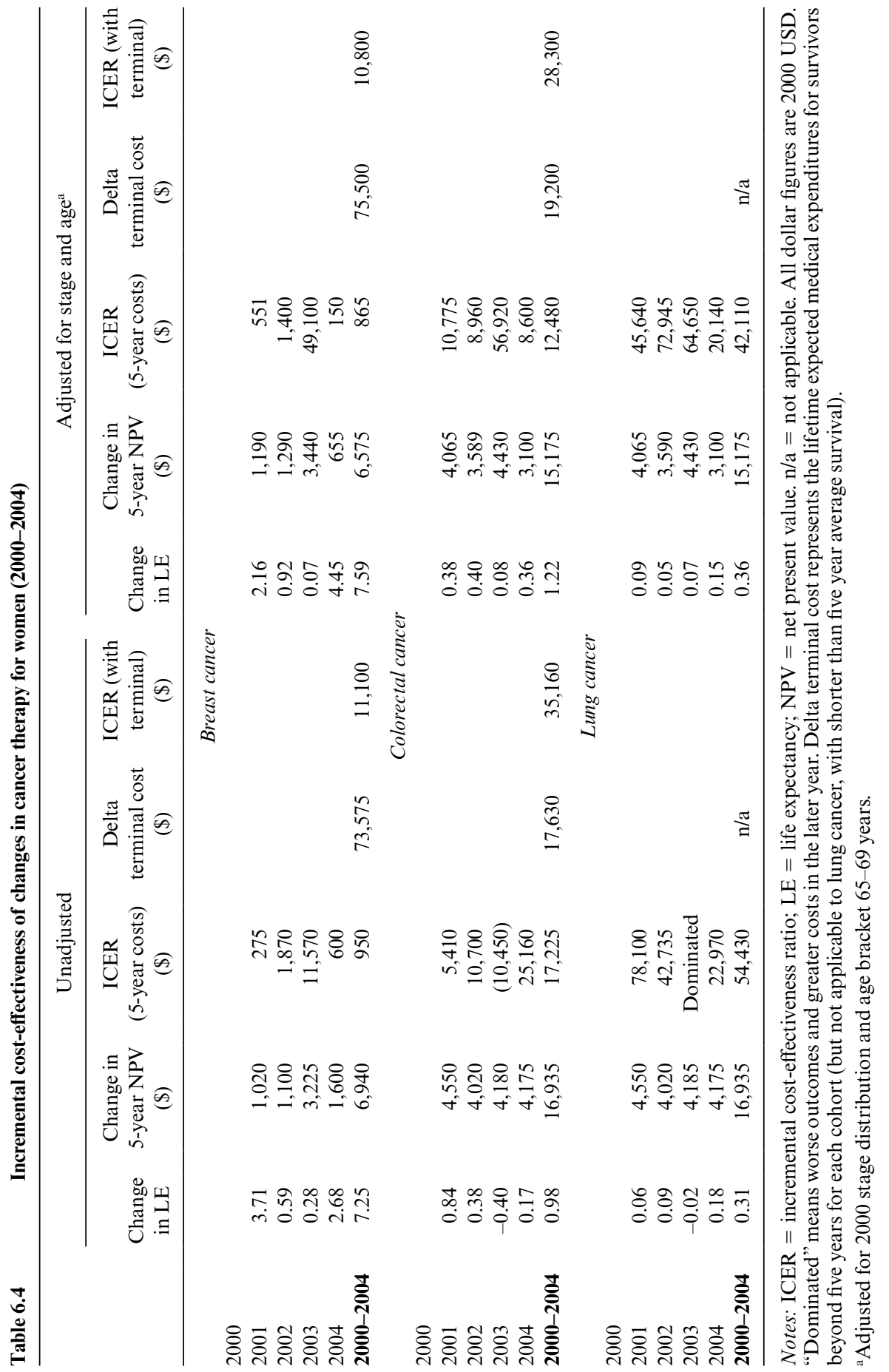


gained when diagnosed in 2004 as compared to 2000. Lung cancer advances lead to the lowest life expectancy improvements, with approximately 2.6 to 4.3 months gained in life expectancy between 2004 and 2000 .

The average life expectancy for breast, lung, and colorectal cancer was greater than the five years of expenditures in the data. Thus, to match medical costs to life expectancy, we estimated a terminal component for medical costs for these cohorts; the difference in terminal costs for medical care between cohorts diagnosed in 2004 and 2000 is shown in a separate column in tables 6.4 and 6.5. Incremental cost-effectiveness ratios were calculated with and without the terminal cost component. Adjusted expenditures increased across all cohorts, resulting in an incremental cost-effectiveness ratio greatest for lung cancer, at $\$ 94,110$ for men diagnosed with lung cancer in 2004 as compared to 2000, adjusted for age and stage of disease. Women with lung cancer were treated at an additional $\$ 42,110$ per life-year gained in 2004 compared with 2000. Colorectal cancer had the next highest marginal cost-effectiveness ratios for treatment advances, with $\$ 54,000$ per life-year gained for men and $\$ 28,300$ per life-year gained for women. The medical care for men with prostate cancer resulted in an adjusted $\$ 10,300$ per life-year gained. Advances in the treatment of women with breast cancer resulted in an additional $\$ 10,800$ per life-year gained when diagnosed in 2004 as compared with 2000. We compared adjusting for both AJCC and historical stage of disease to calculate incremental cost-effectiveness ratios but, given the close similarity of findings, report only AJCC-adjusted estimates in tables 6.4 and 6.5 .

\subsubsection{Stage-Specific Survival Trends}

We also explored stage-specific survival trends between cohorts diagnosed in 2004 and 2000, adjusted for age sixty-five to sixty-nine years in figures 6.11 and 6.12. Improvements in the stage-specific survival rates differed for each cancer cohort. Overall survival improvements were not evenly distributed across stages in most cancer cohorts. Breast cancer survival showed the largest gain for stage 3 disease (14 percent), but essentially no gain for stage 4 disease. Women with colorectal cancer showed a more consistent 4.0 to 4.8 percent increase in survival for stages 2 to 4 disease during this time period. Women and men with stage 1 lung cancer experienced the largest gain in survival-7.7 percent for women and 9.1 percent for men, when diagnosed in 2004 as compared with 2000. For prostate cancer, survival for men with distant disease declined by 2.6 percent, and increased modestly for local and regional disease. Our findings for prostate cancer, breast cancer, and men with lung cancer strongly argue against the Will Rogers phenomenon (see discussion in subsection 6.4.3). 


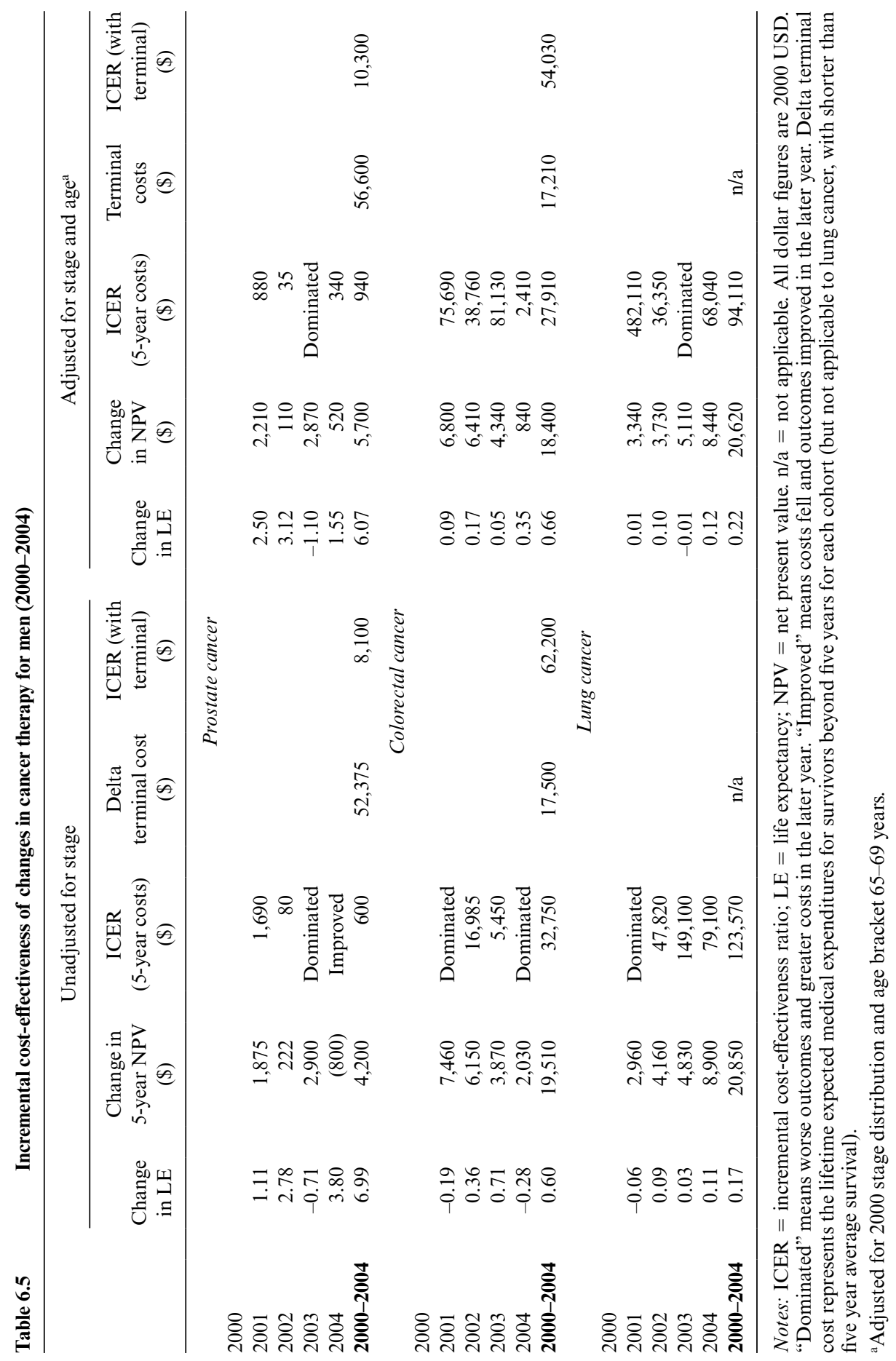



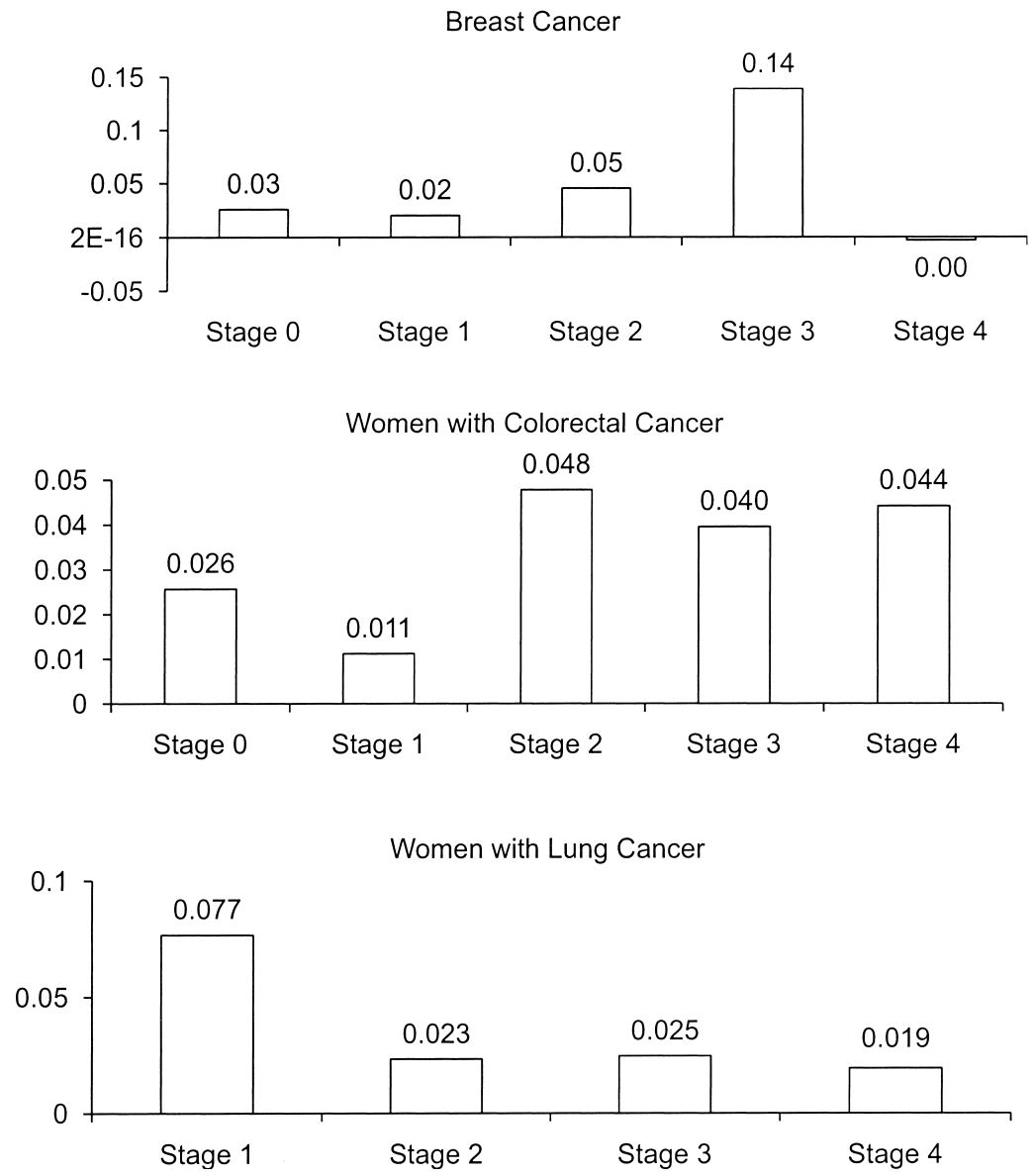

Fig. 6.11 Change in 5-year survival probability (2000-2004) for women by AJCC stage

\subsection{Discussion}

\subsubsection{Summary and Interpretation of Results}

Our analysis suggests that advances in the medical care of cancer patients were not uniformly cost-effective during the periods of analysis, but varied based upon gender and cancer cohort. First, we found that medical progress in the treatment of women with breast and colorectal cancer was generally not cost-effective during the earlier period 1986 to 1994. For women with these cancers, earlier years dominate later ones, since adjusted survival declined while expenditures increased. During the early 2000s, this trend reversed, with advances in treatment for breast cancer now highly cost ef- 

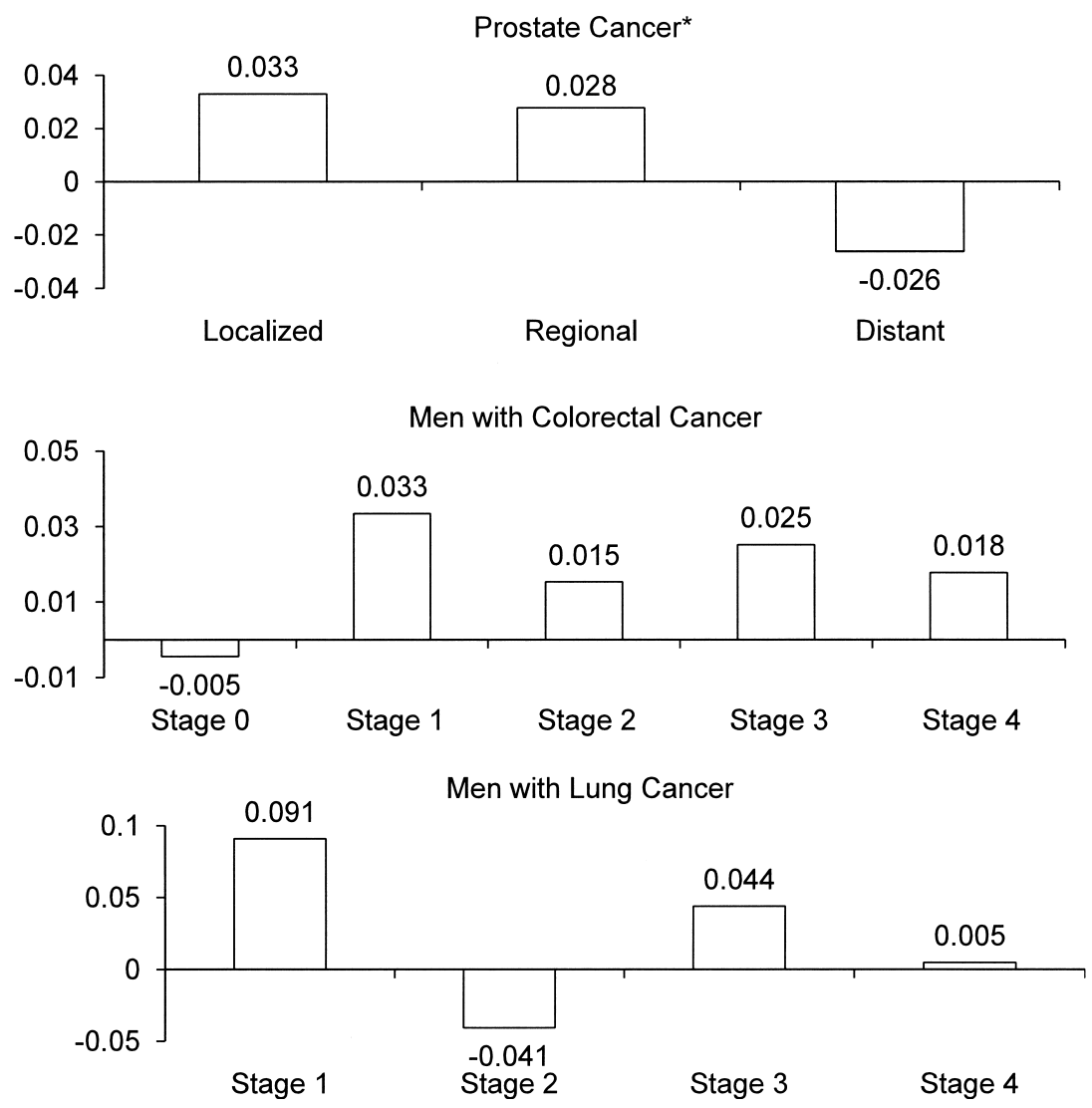

Fig. 6.12 Change in 5-year survival probability (2000-2004) for men by

\section{AJCC stage}

Notes: Prostate cancer* AJCC stages combined stage 1 and 2 for Localized, stage 3 for Regional, and stage 4 for Distant. This was done because the AJCC Cancer Staging Manual Sixth Edition, released in 2003, reclassified almost all previous prostate cancer stage 1 disease into stage 2 disease beginning in 2004 (less than 0.5 percent of prostate cancer patients are classified as stage 1 after 2004).

fective, and advances for women with colorectal cancer slightly less costeffective but still within a generally accepted range for cost per life-year gained (below $\$ 50,000$ to 100,000 per life-year gained-assuming these years were mostly of high quality, which may not necessarily be the case).

The results for men with cancer diagnosed from 1986 to 1994 contrast sharply with the results for women. For prostate cancer, the adjusted costeffectiveness ratios imply that the changes were cost-effective (except in 1989 and 1990, when adjusted survival fell). For some years, like 1992 and 1993 , costs actually fell while survival rose. For men with colorectal cancer, there were highly cost-effective changes in every year between 1986 and 1994. 
Between 1986 and 1994, the stage of breast, prostate, and colorectal cancer at the time of diagnosis among Medicare beneficiaries shifted to more limited, earlier disease. Although we do not address the issue directly, other authors have found that some cancer screening strategies are cost-effective in elderly populations. Our results from this era show that some cancers are diagnosed at earlier stages in Medicare beneficiaries, as would be expected with intensified screening. The findings do not suggest, however, that earlier diagnosis was consistently associated with improvement in the outcomes of treatment. While survival rates for men with prostate and colorectal cancer rose between 1986 and 1994, adjusted survival for women fell even at this time when more screening techniques were being recommended. The change in probability of earlier stage diagnosis was relatively flat for the 2000 to 2004 era, implying small to flat changes in screening rates, as we discuss next.

\subsubsection{Lead and Length-Time Bias}

Lead and length-time bias may contribute to observed survival gains. These biases are well-known to be associated with cancer screening programs (Duffy et al. 2008). Prostate and breast cancer are particularly prone to overdiagnosis - an extreme form of length-time bias - from intensive screening efforts. Prostate cancer screening programs and mammography for breast cancer can increase the diagnosis of indolent, very slow growing, early stage tumors (such as ductal carcinoma in situ, or DCIS breast cancer) which, if left undetected, would have been unlikely to clinically present or lead to death (Yen et al. 2003). Lead-time bias is perhaps most studied in regards to prostate cancer, where estimates of a lead-time of six years for a man diagnosed at age seventy-five is generally accepted for men diagnosed in the PSA-screening era as opposed to the prescreening era (Draisma et al. 2003). During the 2000 to 2005 time period, PSA screening rates have been reported in the Medicare population. Drazer et al. (2011) report modest age-based changes in screening rates between 2000 and 2005. They report that PSA screening rates increased by 3.1 percent (from 43 to 46.1 percent) of the population aged sixty-five to sixty-nine years, 3.8 percent for the population aged seventy to seventy-four years, 0 percent for population aged seventy-five to seventy-nine years, and a dramatic 15 percent increase for the population aged eighty to eighty-five years (Drazer et al. 2011). Overall, we found an age- and stage-adjusted six additional years of life for men diagnosed with prostate cancer in 2004. We find that given the small changes in PSA screening between these years, our results are unlikely to be entirely, or even mostly, accounted for by lead- and length-time bias due to improved prostate cancer screening (Duffy et al. 2008).

Interestingly, the results of our stage-specific survival trends for lung cancer might suggest a role for lead- or length-time bias for men and women diagnosed with stage 1 disease. The five-year probability of survival increased dramatically for both men and women with stage 1 lung cancer (by 
9.1 percent and 7.7 percent, respectively). As lung cancer screening is not a population-based recommendation at this time, we postulate that perhaps this increase in stage 1 disease reflects increased use of CT imaging with an incidental diagnosis of early-stage lung cancer in the population. We do not understand the cause for this finding, which to our knowledge, has not yet been reported.

\subsubsection{Will Rogers Phenomenon}

One possible explanation for our finding of increased stage-adjusted survival among some groups of cancer patients is that they are caused by changes over time in the classification of cancer patients to a more advanced stage even though the underlying disease of patients has not changed over time. The idea is that "up-staging" classifications could move patients with a better prognosis over time to previously poorer prognostic groups. With that move, measured survival for lower stage patients will improve (since these stages no longer include patients who truly have more advanced disease), as will measured survival for higher stage patients (since presumably the patients who are upstaged have less advanced disease than the typical patient who would be diagnosed with advanced cancer regardless of when the staging took place). In the literature, this explanation is called the Will Rogers phenomenon (Feinstein, Sosin, and Wells 1985). Will Rogers once quipped, "When the Okies left Oklahoma and moved to California, they raised the average intelligence level in both states." The Will Rogers phenomenon has particularly been postulated to impact advanced stages of disease (Chee et al. 2008).

Other authors have suggested that long-term historical comparisons of cancer staging may lead to erroneous conclusions regarding survival based on the Will Rogers phenomenon (Gofrit et al. 2008). For example, in the case of prostate cancer, researchers compared grade (Gleason score) classifications from 1990 to 1992 with re-reading by pathologists in 2002 to 2004, blinded to the original readings (Albertsen et al. 2005). They found that the contemporary Gleason scores were significantly higher when read according to 2002 to 2004 pathologic criteria. They estimated that consequently, the contemporary prostate-cancer adjusted mortality rate would be 28 percent lower than standard historical rates without any apparent change in actual outcomes (Albertsen et al. 2005).

Another analysis evaluated the effect of new technology (positron emission tomography, or PET scanning) on stage migration in the post-PET era (1999-2004) and pre-PET (1994-1998) (Chee et al. 2008). The authors found a 5.4 percent decline in patients with stage 3 disease and an 8.4 percent increase in stage 4 disease between these periods. The authors argue that retrospectively, the PET period was associated with a marginally better overall survival (hazard ratio of 0.95 compared with pre-PET survival), which was entirely limited to those with stage 3 (HR 0.77) and stage 4 
(HR 0.64) disease, as opposed to patients with stage 1 or 2 disease. Though we analyze two different eras for stage-adjusted survival within each era, we do not make comparisons between these eras.

The causal pathway for the Will Rogers phenomenon rests mostly on improved sensitivity of imaging modalities over time. If imaging technologies for cancer staging did not improve substantially over time, then it is inappropriate to read the results we present here as consistent with the Will Rogers phenomenon. We believe that the literature on imaging and cancer staging does not support the idea that there have been clinically substantial advances in cancer imaging over this period.

While an extensive literature is devoted to marginal improvements in sensitivity or specificity for cancer staging of imaging advances (Cooper et al. 2011; Gould et al. 2003) - CT-PET over conventional CT, MRI over CT or PET-CT - actual evidence for improved outcomes (survival) with these technologies is nonexistent. In fact, at least one highly publicized study, a randomized trial of combined PET-CT imaging versus conventional imaging enrolled during 2002 through 2007, did not find an effect on overall mortality for the use of the advanced imaging modality (Fischer et al. 2009). Furthermore, higher false positive rates have been documented with PET-CT for lung cancer, which could lead to erroneous conclusions regarding the utility of surgical management (and potential for cure) (Darling et al. 2011). These types of studies demonstrate that better outcomes should not necessarily be assumed simply because technology is next-generation.

In summary, the Will Rogers phenomenon remains an interesting theoretical phenomenon in regards to cancer staging and survival. Yet the weight of evidence does not support that it occurs along time horizons relevant to this analysis. Furthermore, improved outcomes based on advances in today's imaging technologies, as the presumed causal pathway for the Will Rogers phenomenon, is not supported by evidence for superior outcomes based on a review of today's medical literature.

\subsubsection{Competing Risks}

The possibility of competing risks is an important caveat to interpreting our results as directly measuring the cost-effectiveness of technological change in cancer therapy or cancer screening. Patients with cancer are naturally in the age group most at risk of cardiovascular disease. It is plausible that some of our findings for improvements in life expectancy (particularly for prostate cancer patients) occur because of improved care for important competing risks for death, not necessarily due to improvements in cancer treatments. This is particularly true for prostate cancer patients - who even after prostate cancer diagnosis, are still more likely to suffer a death from cardiovascular disease as opposed to prostate cancer-specific causes. There is extensive evidence that heart disease treatment in the population we 
study has results in improved outcomes for patients over the relevant period (Cutler and McClellan 2001; Lichtenberg 2004; Rodriguez et al. 2006; Skinner, Staiger, and Fisher 2006).

Authors publishing based on SEER data have consistently reported 100 percent five-year prostate-cancer-specific survival rates for patients diagnosed with localized disease (National Cancer Institute 2010). However, there is significant difficulty with correctly identifying causes of death in the elderly population. For example, if a patient diagnosed with localized prostate cancer is given androgen deprivation therapy and subsequently dies of a heart attack when androgen deprivation therapy has been shown to increase risk for such cardiac events (D'Amico et al. 2007; Keating, O'Malley, and Smith 2006), does this clearly constitute a death unrelated to prostate cancer?

Lastly, our claim that technological innovations may have been costeffective in some cases requires an assumption that there is a direct causal relationship between the expenditures and outcomes, and that the effects of characteristics that change over time and are not measured in the available data, such as health behaviors, are inconsequential. Though the data sets we use are the richest available national data on cancer patients, we necessarily do not observe every clinical characteristic that may be important. For example, we do not have good observations on adherence to therapy, which plays an important role in determining survival and expenditure outcomes for cancer patients. Also, there may be important trends in unmeasured comorbid conditions, such as obesity. Trends in such characteristics could be responsible for the finding that survival actually worsened over time for women with breast or colorectal cancer; for example, an increasing percentage of women with breast cancer might have had significant comorbidities that influenced survival.

\subsubsection{Younger Populations}

Our findings cannot be presumed to apply to younger cancer patients or to the types of cancers that are more common among the young. However, cancer is primarily a disease of the elderly, and this is particularly true of the types of cancer that we studied. More than two-thirds of all cancer deaths occur among people aged sixty-five and older, and cancer is the second leading cause of death among the elderly, accounting for 113 deaths per year per 10,000 elderly people. Of all cancer patients alive in 2001, 61 percent were aged sixty-five or older.

\subsubsection{Concluding Remarks}

We find that trends in expenditures and outcomes among Medicare beneficiaries strongly suggest that changes in treatment during the periods we studied may have improved outcomes for some but not all cancers evalu- 
ated, and even under favorable assumptions would only be considered costeffective for a subset of cancers. The years of analysis most corresponding to the recent treatment era did show generally cost-effective medical advances for cancer treatment, with the possible exception of men with lung cancer.

The American health care system simultaneously provides incentives for the use of cost-effective and cost-ineffective care. Only by evaluating each technology individually, and the clinical contexts in which it is used, will it be possible to determine whether it is a good investment. Such information is the foundation for rational choices about distributing limited health care resources.

\section{References}

Albertsen, P. C., J. A. Hanley, G. H. Barrows, D. F. Penson, P. D. Kowalczyk, M. M. Sanders, and J. Fine. 2005. "Prostate Cancer and the Will Rogers Phenomenon." Journal of the National Cancer Institute 97 (17): 1248-53.

Bailar, J. C., III, and H. L. Gornik. 1997. "Cancer Undefeated." New England Journal of Medicine 336 (22): 1569-74.

Breen, N., K. A. Cronin, H. I. Meissner, S. H. Taplin, F. K. Tangka, J. A. Tiro, and T. S. McNeel. 2007. "Reported Drop in Mammography: Is This Cause for Concern?" Cancer 109 (12): 2405-59.

Chambers, J. D., and P. J. Neumann. 2011. "Listening to Provenge-What a Costly Cancer Treatment Says about Future Medicare Policy." New England Journal of Medicine 364 (18): 1687-89.

Chee, K. G., D. V. Nguyen, M. Brown, D. R. Gandara, T. Wun, and P. N. Lara Jr. 2008. "Positron Emission Tomography and Improved Survival in Patients with Lung Cancer: The Will Rogers Phenomenon Revisited." Archives of Internal Medicine 168 (14): 1541-49.

Cooper, K. L., Y. Meng, S. Harnan, S. E. Ward, P. Fitzgerald, D. Papaioannou, L. Wyld, C. Ingram, I. D. Wilkinson, and E. Lorenz. 2011. "Positron Emission Tomography (PET) and Magnetic Resonance Imaging (MRI) for the Assessment of Axillary Lymph Node Metastases in Early Breast Cancer: Systematic Review and Economic Evaluation." Health Technology Assessment 15 (4): iii-iv, 1-134.

Cutler, D. 2004. Your Money or Your Life: Strong Medicine for America's Health Care System. New York: Oxford Press.

- 2008. "Are We Finally Winning the War on Cancer?" Journal of Economic Perspectives 22 (4): 3-26.

Cutler, D. M., and M. McClellan. 2001. "Is Technological Change in Medicine Worth It?" Health Affairs (Millwood) 20 (5): 11-29.

D'Amico, A. V., J. W. Denham, J. Crook, M. H. Chen, S. Z. Goldhaber, D. S. Lamb, D. Joseph, et al. 2007. "Influence of Androgen Suppression Therapy for Prostate Cancer on the Frequency and Timing of Fatal Myocardial Infarctions." Journal of Clinical Oncology 25 (17): 2420-25.

Darling, G. E., D. E. Maziak, R. I. Inculet, K. Y. Gulenchyn, A. A. Driedger, Y. C. Ung, C. S. Gu, et al. "Positron Emission Tomography-Computed Tomography Compared with Invasive Mediastinal Staging in Non-Small Cell Lung Cancer: 
Results of Mediastinal Staging in the Early Lung Positron Emission Tomography Trial." Journal of Thoracic Oncology 6 (8): 1367-72.

Draisma, G., R. Boer, S. J. Otto, I. W. van der Cruijsen, R. A. Damhuis, F. H. Schroder, and H. J. de Koning. 2003. "Lead Times and Overdetection Due to Prostatespecific Antigen Screening: Estimates from the European Randomized Study of Screening for Prostate Cancer." Journal of the National Cancer Institute 95 (12): 868-78.

Drazer, M. W., D. Huo, M. A. Schonberg, A. Razmaria, and S. E. Eggener. 2011 "Population-Based Patterns and Predictors of Prostate-Specific Antigen Screening among Older Men in the United States.” Journal of Clinical Oncology 29 (13): $1736-43$.

Duffy, S. W., I. D. Nagtegaal, M. Wallis, F. H. Cafferty, N. Houssami, J. Warwick, P. C. Allgood, et al. 2008. "Correcting for Lead Time and Length Bias in Estimating the Effect of Screen Detection on Cancer Survival." American Journal of Epidemiology 168 (1): 98-104.

Elkin, E. B., and P. B. Bach. 2010. "Cancer's Next Frontier: Addressing High and Increasing Costs." Journal of the American Medical Association 303 (11): 1086-87.

Feinstein, A. R., D. M. Sosin, and C. K. Wells. 1985. "The Will Rogers Phenomenon. Stage Migration and New Diagnostic Techniques as a Source of Misleading Statistics for Survival in Cancer." New England Journal of Medicine 312 (25): 1604-48.

Fischer, B., U. Lassen, J. Mortensen, S. Larsen, A. Loft, A. Bertelsen, J. Ravn, et al. 2009. "Preoperative Staging of Lung Cancer with Combined PET-CT." New England Journal of Medicine 361 (1): 32-39.

Fisher, E. S., J. E. Wennberg, T. A. Stukel, and S. M. Sharp. 1994. "Hospital Readmission Rates for Cohorts of Medicare Beneficiaries in Boston and New Haven." New England Journal of Medicine 331 (15): 989-95.

Fisher, E. S., F. S. Whaley, W. M. Krushat, D. J. Malenka, C. Fleming, J. A. Baron, and D.C. Hsia. 1992. "The Accuracy of Medicare's Hospital Claims Data: Progress Has Been Made, But Problems Remain." American Journal of Public Health 82 (2): $243-48$.

Fleming, I. D. 2001. "AJCC/TNM Cancer Staging, Present and Future.” Journal of Surgical Oncology 77 (4): 233-36.

Freeman, J. L., C. N. Klabunde, N. Schussler, J. L. Warren, B. A. Virnig, and G. S. Cooper. 2002. "Measuring Breast, Colorectal, and Prostate Cancer Screening with Medicare Claims Data.” Medical Care 40 (8 Suppl): IV-36-42.

Fuchs, V. R., and A. M. Garber. 1990. "The New Technology Assessment." New England Journal of Medicine 323 (10): 673-77.

Fuchs, V. R., and A. M. Garber. 2003. "Medical Innovation: Promises and Pitfalls." Brookings Review. http://www.brookings.edu/articles/2003/winter_technology_ fuchs.aspx.

Garber, A. M., and T. MaCurdy. 1993. "Nursing Home Discharges and Exhaustion of Medicare Benefits." Journal of the American Statistical Association 88: 727-36.

Garber, A. M., and C. E. Phelps. 1997. "Economic Foundations of Cost-Effectiveness Analysis." Journal of Health Economics 16 (1): 1-31.

Gofrit, O. N., K. C. Zorn, G. D. Steinberg, G. P. Zagaja, and A. L. Shalhav. 2008. "The Will Rogers Phenomenon in Urological Oncology." Journal of Urology 179 (1): $28-33$.

Gould, M. K., W. G. Kuschner, C. E. Rydzak, C. C. Maclean, A. N. Demas, H. Shigemitsu, J. K. Chan, and D. K. Owens. 2003. "Test Performance of Positron 
Emission Tomography and Computed Tomography for Mediastinal Staging in Patients with Non-Small-Cell Lung Cancer: A Meta-Analysis." Annals of Internal Medicine 139 (11): 879-92.

Hutter, R. L. 1984. "At Last-Worldwide Agreement on the Staging of Cancer." AMA Archives of Surgery 122: 1235-39.

Institute of Medicine. 1977. Reliability of Medicare Hospital Discharge Records. Washington, DC: National Academy of Sciences.

Keating, N. L., A. J. O'Malley, and M. R. Smith. 2006. "Diabetes and Cardiovascular Disease during Androgen Deprivation Therapy for Prostate Cancer." Journal of Clinical Oncology 24 (27): 4448-56.

Lenzer, J. 2006. "US Cancer Mortality Falls for the First Time." British Medical Journal 332 (7539): 444.

Lichtenberg, F. R. 2004. "Sources of U.S. Longevity Increase, 1960-2001.” Quarterly Review of Economics and Finance 44:369-89.

Mark, D. H. 1994. "Mortality of Patients after Radical Prostatectomy: Analysis of Recent Medicare Claims." Journal of Urology 152 (3): 896-98.

McBean, A. M., J. L. Warren, and J. D. Babish. 1994. "Measuring the Incidence of Cancer in Elderly Americans Using Medicare Claims Data." Cancer 73 (9): 2417-25.

Medicare Trustees. 2008. The 2008 Annual Report of the Boards of Trustees of the Federal Hospital Insurance and Federal Supplementary Medical Insurance Trust Funds. Baltimore, MD: C. f. M. M. Services.

Meltzer, D. 2003. "Can Medical Cost Effectiveness Analysis Identify the Value of Medical Research?" In Measuring the Gains from Medical Research: An Economic Approach, edited by Kevin M. Murphy and Robert H. Topel, 206-247. Chicago: University of Chicago Press.

Murphy, K. M., and R. H. Topel. 2003. "The Economic Value of Medical Research.” In Measuring the Gains from Medical Research: An Economic Approach, edited by Kevin M. Murphy and Robert H. Topel, 41-73. Chicago: University of Chicago Press.

- 2005. "Black-White Differences in the Economic Value of Improving Health.” Perspect Biol Med 48 (1 Suppl): S176-94.

National Cancer Institute. 2010. "Cancer Trends Progress Report-2009/2010 Update." Accessed on May 24, 2010. http://progressreport.cancer.gov.

Owens, D. K., A. Qaseem, R. Chou, and P. Shekelle. 2011. "High-Value, CostConscious Health Care: Concepts for Clinicians to Evaluate the Benefits, Harms, and Costs of Medical Interventions." Annals of Internal Medicine 154 (3): 174-80.

Porter, M. E. 2010. "What Is Value in Health Care?” New England Journal of Medicine 363 (26): 2477-81.

Potetz, L., and L. F. DeWilde. 2009. Cancer and Medicare: A Chartbook. Cancer Action Network: American Cancer Society.

Rodriguez, T., M. Malvezzi, L. Chatenoud, C. Bosetti, F. Levi, E. Negri, and C. La Vecchia. 2006. "Trends in Mortality from Coronary Heart and Cerebrovascular Diseases in the Americas: 1970-2000." Heart 92 (4): 453-60.

Siegler, M., A. Weisfeld, and D. Cronin. 2003. "Is Medical Research Cost Effective? Response to Murphy and Topel." Perspectives in Biology and Medicine 46 (3 Suppl): S129-37.

Skinner, J. S., D. O. Staiger, and E. S. Fisher. 2006. "Is Technological Change in Medicine Always Worth It? The Case of Acute Myocardial Infarction." Health Affairs (Millwood) 25 (2): w34-47.

Yen, M. F., L. Tabar, B. Vitak, R. A. Smith, H. H. Chen, and S. W. Duffy. 2003. 
"Quantifying the Potential Problem of Overdiagnosis of Ductal Carcinoma in situ in Breast Cancer Screening." European Journal of Cancer 39 (12): 1746-54.

\section{Comment Amitabh Chandra}

It is always slightly terrifying to discuss a chapter by a team with so much intellectual firepower, and in this case doubly so, for Jay Bhattacharya and Alan Garber have taught me so much about health economics. My comments on their work will focus on the broader questions about assessing the productivity of medical spending, be it on cancer or other diseases.

In this chapter the authors demonstrate that the distribution of benefits from medical progress in cancer is not egalitarian. They find that spending on women with breast and colorectal cancer was not cost-effective until the mid-1990s, but started to look remarkably cost-effective after that; a finding that will excite cancer researchers and their advocates everywhere. The earlier period may even have been harmful as survival fell while expenditures increased. In contrast to the results for women, spending on prostate cancer is shown to confer immensely cost-effective benefits.

I have two comments. The first is one that Jonathan Skinner and I make in our paper "Technology Growth and Expenditure Growth in Healthcare" (Chandra and Skinner 2011). Studies of the aggregate productivity of health care spending collapse costs and benefits across technologies to measure the productivity of spending. The chapter by Bhattacharya and colleagues utilizes this framework, as does David Cutler and Murphy and Topel (2006), who estimate an increase in the value of health roughly three times accumulated health care costs during 1970 to 2000 . Similarly, Lakdawalla et al. (2010) found high average cost-effectiveness for cancer treatments. A close cousin of this approach is found in the considerable work on geographic variations in spending, where health outcomes are regressed on spending. But in this research (which includes a lot of mine), the returns reflect the weighted means of survival gains and costs across different types of treatments. So it could easily be the case that one treatment is responsible for the bulk of the spending and another for the majority of the survival improvements. And while that does not change the overall conclusion about the cost-effectiveness of medical spending, it certainly changes how sanguine

Amitabh Chandra is economist and professor of public policy at the Harvard Kennedy School of Government, a research fellow at the IZA Institute in Bonn, Germany, and a research associate of the National Bureau of Economic Research.

For acknowledgments, sources of research support, and disclosure of the author's material financial relationships, if any, please see http://www.nber.org/chapters/c12440.ack. 